\title{
A Statistical Method for Estimating Catalog Completeness Applicable to Long-Term Nonstationary Seismicity Data
}

\author{
by Amir Hossein Hakimhashemi and Gottfried Grünthal
}

\begin{abstract}
Methods for analyzing the completeness of earthquake historical catalogs are applied to estimate which part of a historical catalog likely includes all earthquakes of a certain magnitude class after a certain time in a given area. Current methods of catalog completeness estimation are not sufficient in case of long-term nonstationary seismicity data. Such methods typically require model presumptions, whereas the method described in this study does not. We develop a statistical approach based on a deviation criterion, which is applicable to stationary, nonstationary, and even multimodal nonstationary seismicity rates. The significance of the method is tested using synthetic catalogs extracted from stationary (exponential based on a Poisson process) and nonstationary (mixed bimodal Weibull-Weibull, gamma, and log-normal) distributions. The method is applied to different parts of the EuroMediterranean region based on long-term seismicity data.
\end{abstract}

\section{Introduction}

The aim of testing the completeness of long-term seismicity data is to determine a highly reliable estimate of the earliest time at which all earthquakes are included in the catalog for different magnitude classes in a given area. However, it is a fact that early historical data are incomplete. The catalog completeness is crucial for estimating reliable seismicity rates and, consequently, for use in seismic hazard assessments. The latter especially require seismicity data from as long of a time period as possible. Such data are often difficult to analyze with respect to their completeness. This is even more problematic in cases of nonstationary seismicity. In this study we introduce a new effective statistical method to test and analyze the catalog completeness. This method is simple and applicable not only for stationary but also for nonstationary long-term seismicity, without considering any presumptions (e.g., any predefined statistical distribution or process, such as a Poisson process) for the seismicity. Long-term seismicity is defined in this study as including one or two millennia of Euro-Mediterranean data.

The frequency of earthquakes in the magnitude classes of the largest events is usually so sparse that statistical methods fail to reliably estimate their completeness time. In such cases, historical methods can be applied (Stucchi et al., 2004). These methods use cultural-historical aspects to re-examine whether certain historical events have a high probability of being included in the chronicles.

Two commonly used methods to determine earthquake catalog completeness are (1) a model by Stepp (1972) and (2) a slope method. The method suggested by Stepp (1972) estimates the completeness time from the standard deviations of empirical annual occurrence rates of events of different strength classes for various time intervals and checks the homogeneity of the graphs with time. The slope method is based on plots of cumulative numbers of earthquakes of a magnitude class with time (in this case, plots of the cumulative energy or strain release have also been used) and estimates the completeness of the records beginning with the time at which the slopes of cumulative plots for events of a given magnitude class show an abrupt or significant gradual change with time. This method is very simple but rather robust (see Grünthal et al., 1998). These two standard methods usually can be applied for stationary seismicity. Even for certain cases of nonstationary seismicity, the slope method can efficiently estimate the completeness time, especially alongside historiographic information.

Albarello et al. (2001) have given an overview of different methods of catalog completeness (Stepp, 1972; Caputo and Postpischl, 1974; Båth, 1983; Mulgaria and Tinti, 1985; Tinti and Mulgaria, 1985a,b; Mulgaria et al., 1987a,b; Rotondi et al., 1994) and suggest a method that tests differences in occurrence rates of events in different time intervals, using a binomially distributed random variable. The completeness probability is then calculated using a conditional probability. The power of the test is examined using only stationary synthetic catalogs (Poisson process). Although the method has been proposed to be appropriate to test the completeness with no predefined conditions, there are some assumptions that are required due to the calculation of the completeness probability but that are not always provided by the data. For example, the probability of completeness, given a certain time span, is assumed to be binomially distributed with the 
distribution parameter equal to 0.5 . It is usually not proven that this precondition is fulfilled.

Rotondi and Garvaglia (2002) introduced a method using a change point process. They assume that the earthquake interevent times follow an exponential distribution under a Poisson process (i.e., it is not appropriate for nonstationary cases). Under this assumption, the problem of the estimation of the completeness time is reduced to a problem of finding the time within which the average occurrence rate of earthquakes changes. Furthermore, although this method may statistically seem more reliable, it is neither simple to apply, nor significantly applicable for cases without plain change points (or times) between complete and incomplete parts of the catalog.

Apart from the simple slope method for stationary seismicity data, the lack of appropriate and robust methods for both stationary and nonstationary data is obvious. For that reason, we describe a new effective method to analyze the catalog completeness that is appropriate for both stationary and nonstationary long-term seismicity data. This method does not contain presumptions (such as any type of statistical distribution of seismicity) so therefore is suitable for any sort of long-term seismicity. The reliability of the new method has been examined by synthetic catalogs extracted from stationary and nonstationary processes. The applicability of the method is presented especially for complicated cases of real earthquake data sets. Although the new method is significant from the statistical point of view and produces reliable results, the natural (aleatory) uncertainties coupled to historical earthquake data make it necessary to apply other appropriate methods (e.g., the slope method) parallel to the new method. The problem of completeness times should not be confused with the determination of the completeness magnitude, $M_{\mathrm{c}}$, which defines the lower threshold magnitude of an earthquake catalog, including a large number of instrumental events that are usually of low magnitudes. $M_{\mathrm{c}}$ is defined as the lowest magnitude, which is defined using the Gutenberg-Richter law displayed in a logarithmic scale (see Wiemer and Wyss, 2000; Cao and Gao, 2002; Woessner and Wiemer, 2005; Amorese, 2007; Mignan et al., 2011).

\section{The Method}

The main idea of the new method is to monitor the variability of the changes (known as deviation in statistics) of the earthquake interevent times in a catalog. As long as these changes are extreme, the earthquake data set is not complete. As soon as the changes decrease and become more stable, the catalog can be considered reasonably complete. In order to quantify the changes in the deviation of the mean value of interevent times, a deviation parameter is needed. This parameter can be investigated by either the standard deviation or the variance of interevent times in any given earthquake data set. The complete part of a data set starts when the deviation parameter remains stable (i.e., when it does not significantly change with time). As no prior distribution has been considered for the earthquake interevent times, the only parameter to be monitored is the deviation parameter.

The method can be applied to cases with timeindependent, time-dependent, and even more complex (such as multimodal or nonparametric) earthquake occurrence rates, because no mean value of earthquake interevent times is considered to test the completeness. In this study, we consider the sample variance of the interevent times as the deviation parameter.

Consider the set of different time spans as

$$
\Theta=\left\{T_{i} \mid i=2, \ldots, n\right\}
$$

with

$$
T_{i}=T-\left\{t_{n-j} \mid j=0, \ldots, n-i\right\}(i=2, \ldots, n),
$$

where $T$ is the set of all earthquake interevent times, and $t_{i}$ is the $i$ th ranked interevent time based on occurrence times. Now define

$$
V_{i}=\frac{\sum_{t \in T_{i}}\left(t-\bar{T}_{i}\right)^{2}}{n_{i}-1}, \quad \bar{T}_{i}=\frac{\sum_{t \in T_{i}} t}{n_{i}}, \quad i=2, \ldots, n
$$

as the unbiased estimation of the sample variance of the set $T_{i}$, where $n_{i}$ is the number of interevent times in the set $T_{i}$.

The variances of $V_{i}$ can be calculated using

$$
\operatorname{Var}\left(V_{i}\right)=\left[E\left(V_{i}\right)\right]^{2}\left(\frac{2}{n_{i}-1}+\frac{\kappa_{i}}{n_{i}}\right),
$$

where $\operatorname{Var}\left(V_{i}\right)$ and $E\left(V_{i}\right)$ are the corresponding variance and statistical expectation of $V_{i}$, respectively (Navidi, 2006). $\sqrt{\operatorname{Var}\left(V_{i}\right)}$ is an estimator for $\sigma$ (standard deviation) of $V_{i} . \kappa$ is the distribution kurtosis (see Joanes and Gill, 1998), which can be calculated either as the sample kurtosis by

$$
\kappa_{i}=\frac{\frac{1}{n_{i}} \sum_{t \in T_{i}}\left(t-\bar{T}_{i}\right)^{4}}{\left[\frac{\left(n_{i}-1\right)}{n_{i}} V_{i}\right]^{2}}-3
$$

or as a population kurtosis using

$$
\begin{aligned}
\kappa_{i}= & {\left[\frac{\left(n_{i}+1\right) n_{i}}{\left(n_{i}-1\right)\left(n_{i}-2\right)\left(n_{i}-3\right)}\right]\left[\frac{\sum_{t \in T_{i}}\left(t-\bar{T}_{i}\right)^{4}}{V_{i}^{2}}\right.} \\
& \left.-\frac{3\left(n_{i}-1\right)^{2}}{\left(n_{i}-2\right)\left(n_{i}-3\right)}\right] .
\end{aligned}
$$

In the next step, the difference between two successive sample variances are calculated as

$$
D V_{i}=V_{i+1}-V_{i}, \quad i=2, \ldots, n .
$$

Finally, the $D V_{i}$ will be monitored against occurrence times. The completeness of the catalog can then be defined as the time in which $D V_{i}$, and consequently $V_{i}$, begin to show a uniform trend. The advantage of selecting $T_{i}$ as in equation (2) is that we can monitor the changes in variances 
while adding each individual event to the current set of events. This gives us the chance to check the completeness starting from the end of the catalog by adding unique events in each step $i$.

Figure 1 illustrates how the new method works using two synthetic catalogs, each extracted from an exponential distribution with the same parameter for the statistical distribution of interevent times (i.e., the time between two successive events). The gray lines represent the normalized cumulative number of events, which are directly interpreted in the classical slope method. In Figure 1a and Figure 1b, respectively, $80 \%$ and $40 \%$ of the events before the given completeness time (here around time 0 on a relative scale) are randomly removed from the catalog. Here it should be mentioned that the completeness time of both catalogs is approximately, but not exactly, time 0 , because the interevent times are randomly produced and therefore the exact completeness time cannot be introduced. The black lines show normalized $V_{i}$ versus time (arbitrary unit). The large change in the trend of $V_{i}$ in Figure 1a explicitly indicates that the complete part starts at a time of about -70 (arbitrary unit). In contrast, in Figure 1b, the complete part is not distinguish-
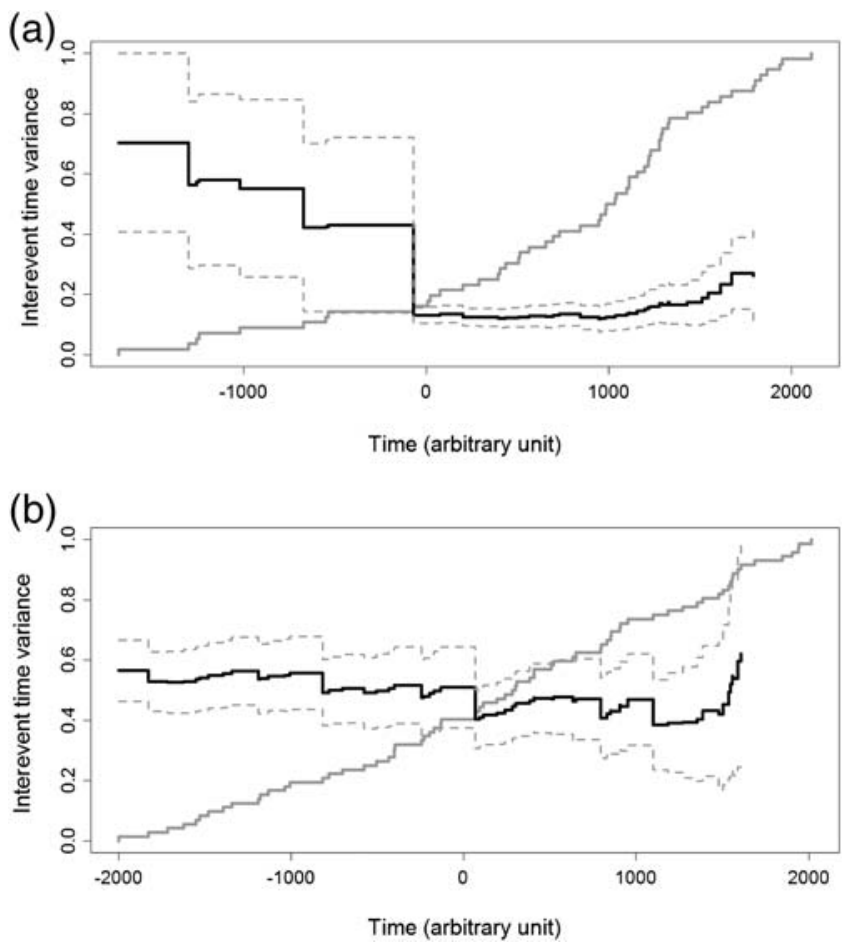

Figure 1. The new method for two different cases of synthetic catalogs. Completeness times for both cases start around time 0 . Solid gray lines show the normalized cumulative number of events. Black lines are the sample variances, $V_{i}$, calculated by equation (3). Dashed gray lines represent the uncertainties coupled to the calculation of $V_{i}$, estimated by $V_{i} \pm \sqrt{\operatorname{Var}\left(V_{i}\right)} \approx V_{i} \pm \sigma$ (see equation 4). Part (a) shows a clear completeness time start at about -70 , with a uniform trend of $V_{i}$ after this time and a significantly nonuniform trend before it. In (b), a completeness time is not distinguishable. able according to the trend of $V_{i}$. In fact, many cases of real historical earthquake catalogs present trends similar to Figure $1 \mathrm{~b}$, where the uniform trend of $V_{i}$ is not explicitly detectable. Therefore, an additional statistical test is needed in order to determine the complete part by characterizing the uniform and nonuniform trends of $V_{i}$. For this reason, the completeness times are determined using two statistical tests, a parametric and a nonparametric test. The parametric $F$-test is applied for testing the homogeneity of consecutive sample variances (Fisher, 1918) of interevent times (i.e., consecutive $V_{i}$ ), where the $F$-test p-value is calculated for each occurrence time.

The calculated p-values are monitored reversely in time, and the completeness time is defined as the first time that the corresponding $\mathrm{p}$-value is larger than an arbitrary confidence probability. This confidence probability can vary. Figure 2 illustrates the application of the $F$-test to the two synthetic cases of Figure 1a and Figure 1b. The confidence probability has been chosen as 0.75 for both cases. As in Figure 1a, the completeness time (about -70) can easily be selected for Figure 2a. Comparing Figures $1 \mathrm{~b}$ and $2 \mathrm{~b}$, the detection of the completeness time is only possible using the $F$-testp-values.

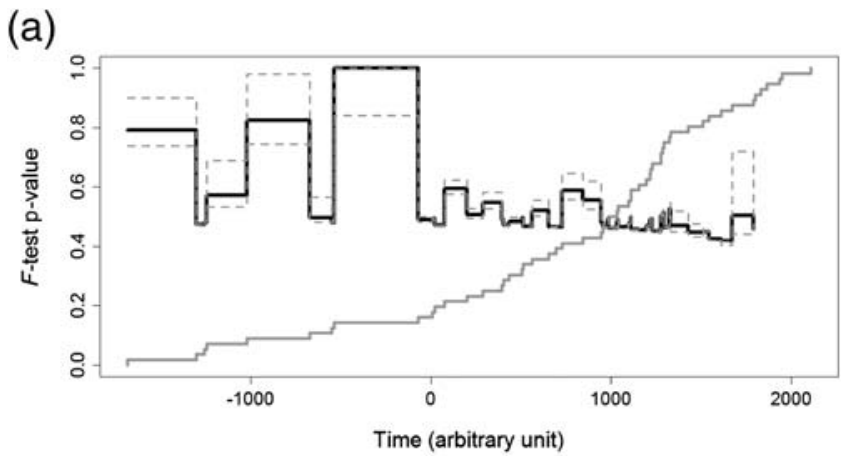

(b)

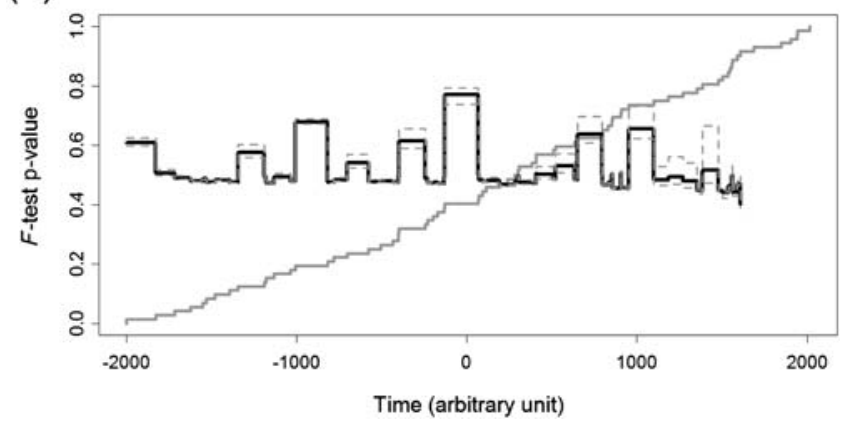

Figure 2. The process of the selection of completeness times for the same cases as in Figure 1. The p-values of the $F$-tests (black lines) are monitored reversely in time, and the completeness time is selected as the first time when the corresponding p-value is larger than a given confidence probability (here, 0.75). The gray lines show the normalized cumulative number of events (same as in Fig. 1). The dashed gray lines demonstrate the $F$-test p-values of $V_{i} \pm \sqrt{\operatorname{Var}\left(V_{i}\right)} \approx V_{i} \pm \sigma$, representing the uncertainties of the p-values. Considering the p-values above 0.75 , the completeness times can easily be selected in both cases. 
The detection of completeness times using the $F$-test for homogeneity of consecutive sample variances, $V_{i}$, is very simple to apply. As with other statistical methods, it requires a sufficient number of events, which can vary depending on the statistical distribution of interevent times. However, it is obvious that an increased amount of high-quality input data results in a higher accuracy of selected completeness time. One issue that should be considered when using the $F$-test is its assumption that the corresponding interevent times are normally distributed. This assumption is not always correct. For this reason, we also apply a nonparametric (NP) test for detection of the inequality in variances. We chose the Siegel-Tukey test (Siegel and Tukey 1960, Lehmann 2006), a version of the Wilcoxon-Mann-Whitney test. The detection of completeness times using the NP test follows the same procedure as for the $F$-test. For the NP test, we consider a higher confidence probability equal to 0.9 , because this test has more sensitive p-values than does the $F$-test. Figure 3 shows the result for the synthetic catalogs in Figure 1. Using the NP test, the completeness times around time 0 can be confirmed.

Also apparent in Figure 1 is an increase of variances after time 1500 , although in this time span the catalog is assumed to be complete. A general reason for this behavior can be the decrease in the number of interevent times, which results in an increase of the variances of the interevent times. Furthermore, one can observe that having fewer than 10
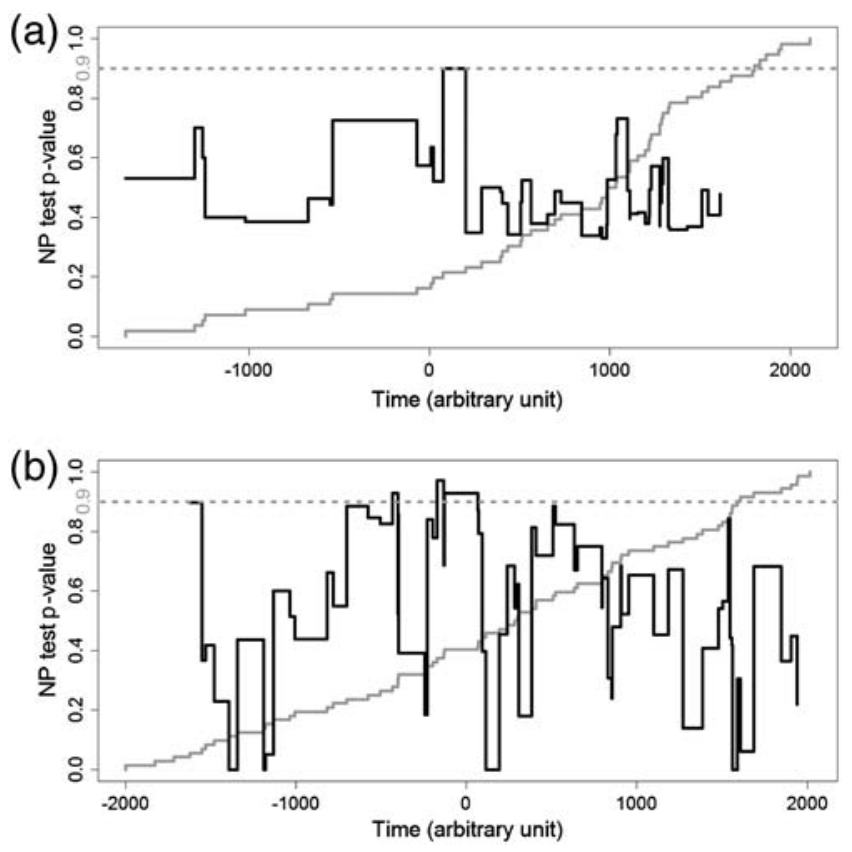

Figure 3. The process of the selection of completeness times for the same cases in Figures 1 and 2. p-values of the nonparametric (NP) test (black lines) are monitored reversely in time, and the completeness time is selected as the first time when the corresponding $\mathrm{p}$-value is larger than a given confidence probability (here, 0.9 ). The gray lines show the normalized cumulative number of events (same as in Figs. 1 and 2). interevent times can result in an increase of the variances. However, if the interevent times are well distributed, the variances should remain stable. It can be concluded that the minimum number of required data to obtain a stable behavior of variances cannot be a rule and depends directly on the distribution of the corresponding interevent times.

\section{Test of the New Method Using Synthetic Catalogs}

In this section, the power of the new method is examined using synthetic catalogs. These catalogs are extracted from different statistical distributions with different characteristics, including (1) an exponential distribution (under a Poisson process) representing a time-independent occurrence rate, (2) a lognormal and a gamma distribution, symbolizing time-dependent occurrence rates, and (3) a bimodal mixed Weibull-Weibull distribution characterizing both timedependent and bimodal occurrence rates (see Figs. 4 and 5).

For the first step, the synthetic catalogs have been built up in a time span between time units from -2000 to 2000 such that, for each distribution, 2000 catalogs are produced by random generation of interevent times. Next, events before the given completeness time (around time 0) have been randomly deleted (with a probability of 0.8 ) from each catalog.

The completeness time of each catalog has been selected automatically by applying the $F$-test for homogeneity of sample variances of interevent times as explained previously in The Method section. The completeness times were selected as the first times (reverse time), when the corresponding p-values are less than the given confidence probability (here 0.8 ). The power of the completeness test has been calculated using the proportion of the number of correctly detected completeness times (after time 0 ) divided by the total number of generated catalogs. The calculated power of the test is about 0.70 for the exponential distribution, 0.77 for the lognormal distribution, 0.71 for the gamma distribution, and 0.69 for the bimodal mixed Weibull-Weibull distribution. A conservative power of the test (i.e., the two-sided number of estimated completeness times between times -200 and 200 , or $10 \%$ of the whole period, divided by the total number of catalogs) has been calculated for all synthetic catalogs. The conservative power of the test is about 0.45 for the exponential distribution, 0.29 for the lognormal distribution, 0.35 for the gamma distribution, and 0.40 for the bimodal mixed Weibull-Weibull distribution (Fig. 4). This shows a significant level of confidence of the new method.

For the next step, a similar procedure is repeated, but the completeness times are selected using the p-value of the NP test instead of the $F$-test p-value. Using each distribution in the same time period (between -2000 and 2000), 2000 synthetic catalogs are produced, but, for this step, only $75 \%$ of the events before time 0 are deleted from each catalog.

As mentioned previously in this article, the confidence probability is considered as 0.9 . The calculated power of the test is about 0.90 for the exponential and the bimodal mixed 
(a)

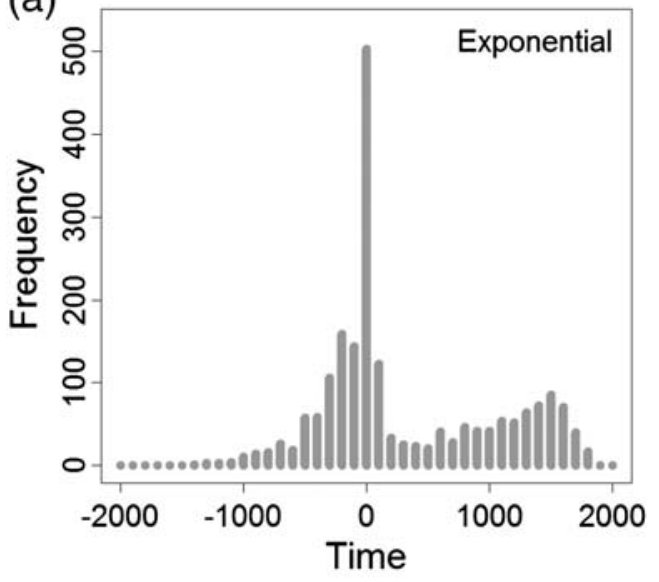

(c)

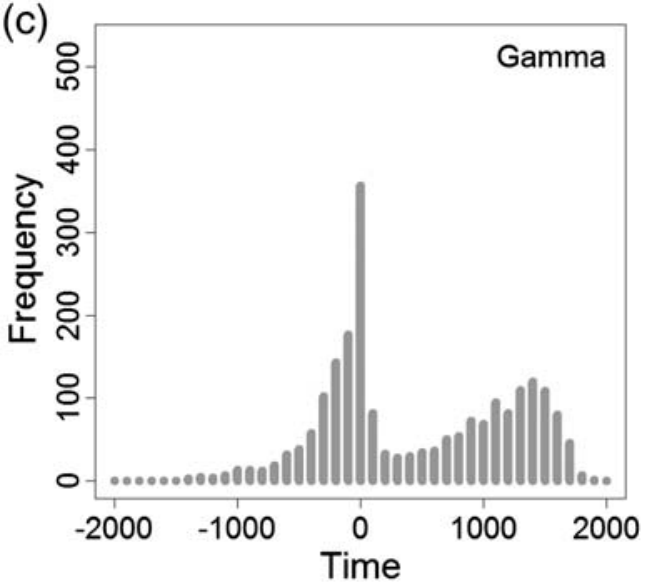

(b)

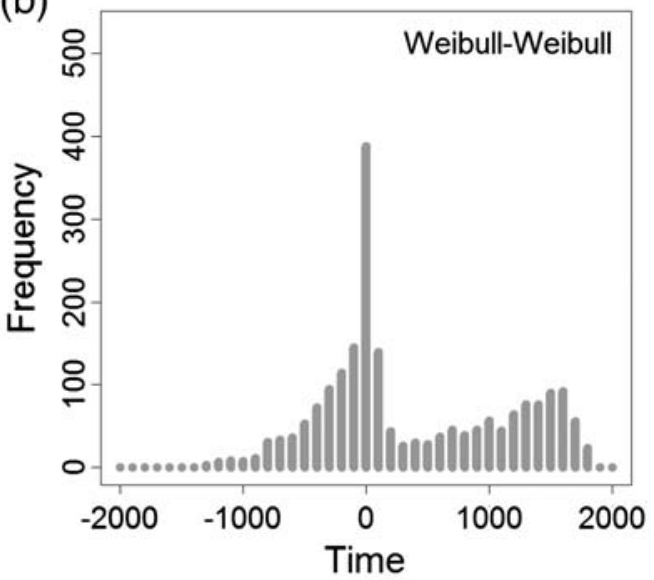

(d)

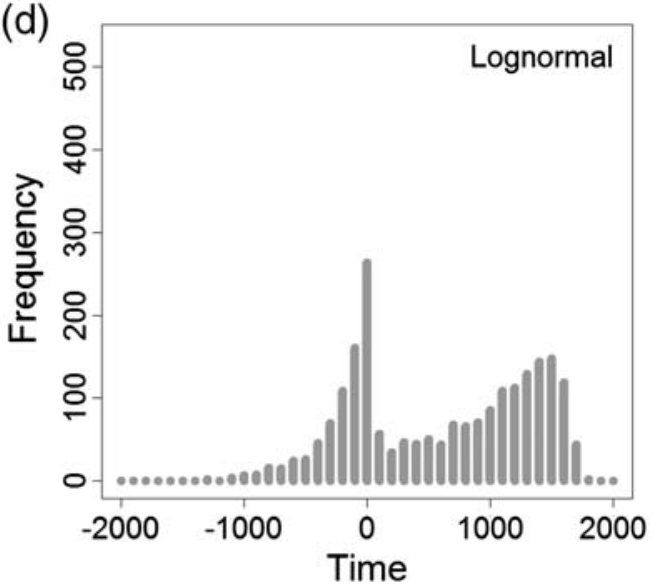

Figure 4. Results of the estimated catalog completeness for more than 2000 synthetic catalogs based on exponential, mixed WeibullWeibull, gamma, and lognormal distributions for the interevent times. The figures show the frequencies of automatically selected completeness times using the $F$-test p-values described in the text. The completeness times have been set at time 0 .

Weibull-Weibull distributions, 0.97 for the lognormal distribution, and 0.96 for the gamma distribution. The conservative power of the test is about 0.21 for the exponential distribution, 0.13 for the lognormal distribution, 0.18 for the gamma distribution, and 0.16 for the bimodal mixed Weibull-Weibull distribution (Fig. 5). This also shows a significant level of confidence of the new method.

In the next step, the power of the method is examined for more complex cases in which fewer events are deleted from the incomplete part of the catalogs. The exponential distribution is used to build up synthetic catalogs for two different cases, a case with $25 \%$ and a case with $50 \%$ deleted events before time 0 . The time periods remain the same between -2000 and 2000.

The p-value of the NP test is used to detect the completeness times. The confidence probability remains in the level of 0.9. The calculated power of the test is about 0.61 for the case of $25 \%$ and 0.71 for the case of $50 \%$ deleted events, respectively. The conservative power of the test is about 0.1 for the case of $25 \%$ and 0.16 for the case of $50 \%$ deleted events. The results show the consistency of the method. For the case of 50\% deleted events, the method is still applicable, because the maximum number of detected completeness times is located around time 0 (see Fig. 6b). For the case of $25 \%$ deleted events, the maximum number of the detected completeness times is not significantly distinguishable. In this case the test fails.

It should be mentioned that the completeness times for the synthetic catalogs were derived on the basis of a completely self-regulating process with fixed and nonflexible confidence probabilities. For single instances of real seismicity data sets, the completeness times can be selected more reliably using additional knowledge about the data set (such as completeness times of other magnitude classes in the same completeness zone, the outcome from neighboring regions, or historical evidence).

\section{Application to Real Long-Term Earthquake Catalog Data}

Here, the method is applied to the data of the EuropeanMediterranean Earthquake Catalogue (EMEC; Grünthal and Wahlström, 2012), which is a spatial and temporal extension 

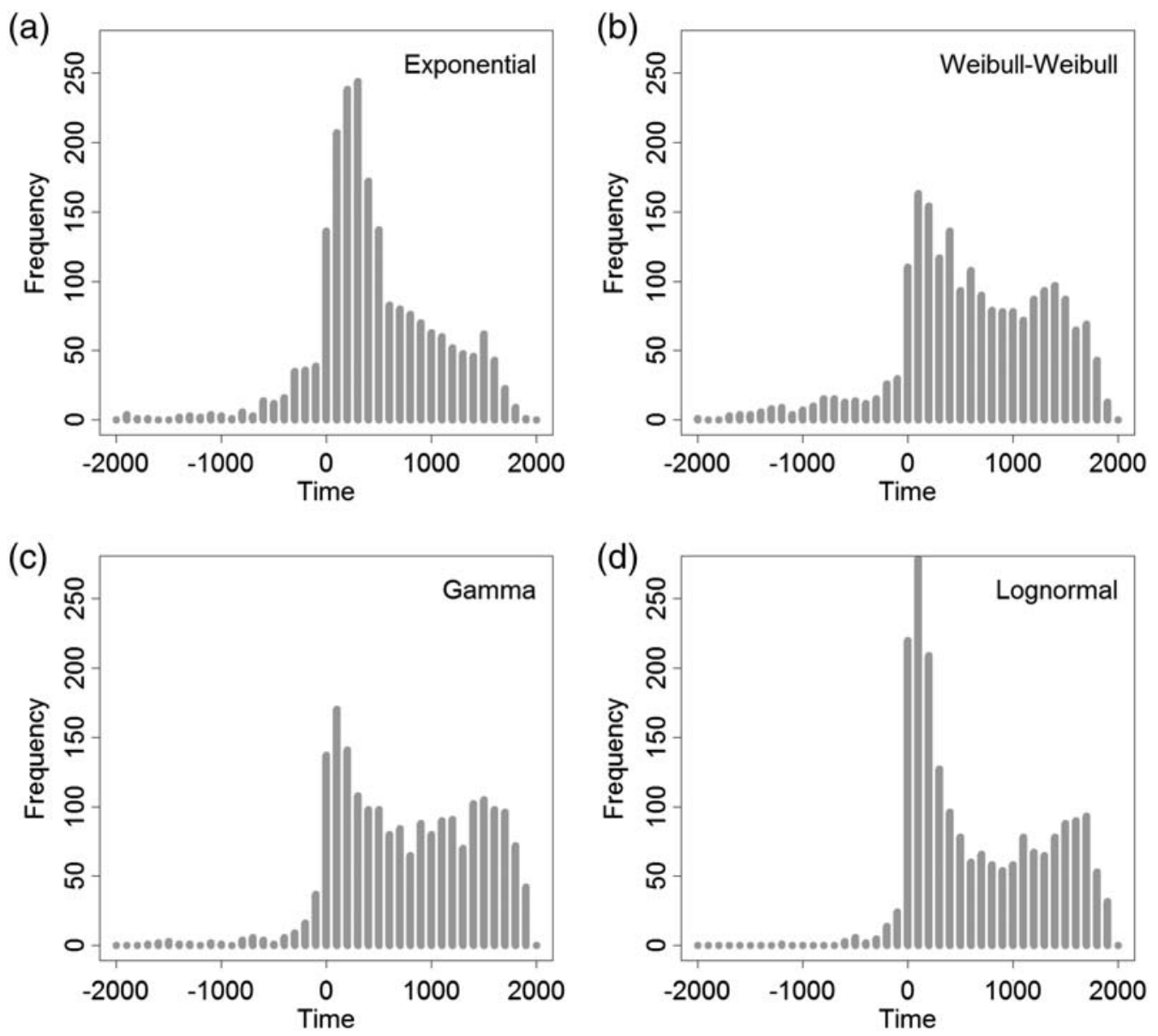

Figure 5. Results of the estimated catalog completeness for more than 2000 synthetic catalogs based on exponential, mixed WeibullWeibull, gamma, and lognormal distributions for the interevent times. The figures show the frequencies of automatically selected completeness times using the p-values of the NP test described in the text. The completeness times have been set at time 0 .
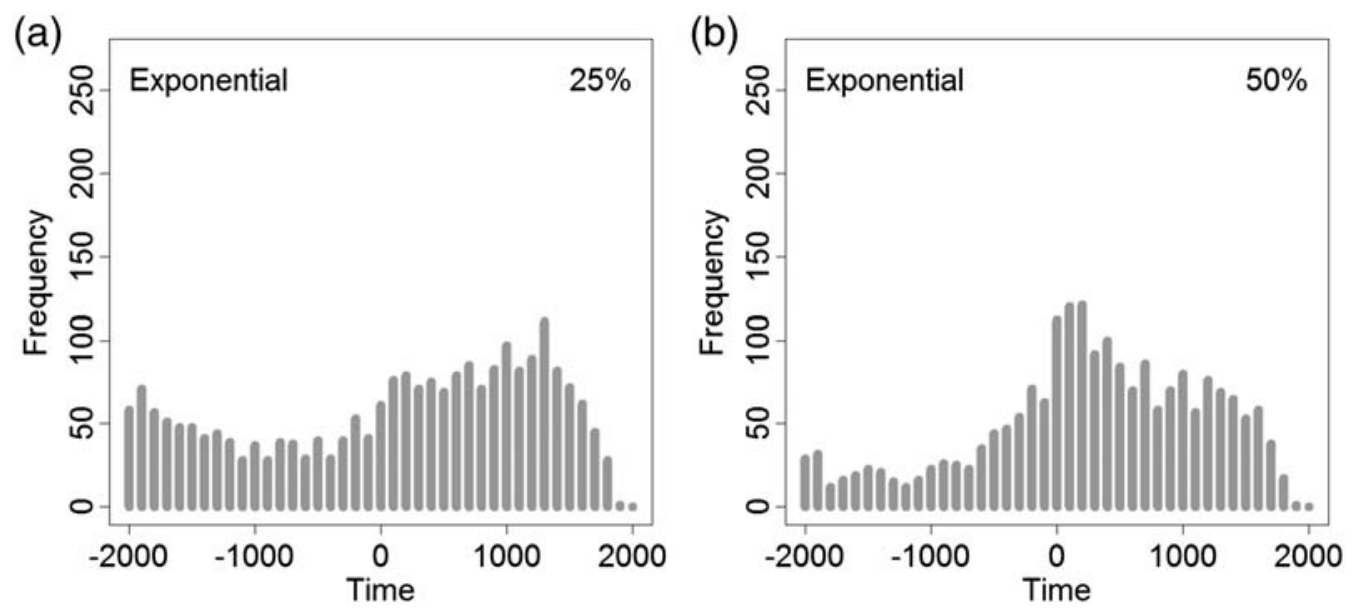

Figure 6. Results of the estimated catalog completeness for more than 2000 synthetic catalogs based on exponential distribution for two cases of (a) $25 \%$, and (b) $50 \%$ of deleted events before time 0 . The figures show the frequencies of automatically selected completeness times using the p-values of the NP test at the level of 0.9 (confidence probability) described in the text. The completeness times have been set at time 0 .

of the unified catalogue of earthquakes in central, northern, and northwestern Europe (CENEC), north of $44^{\circ} \mathrm{N}$ (Grünthal et al., 2009). Completeness analyses of earthquake catalogs should be done considering several issues: (1) the strong spatial differences, (2) how the databases have been constructed according to the availability of historical information, 
(3) how the databases are generated, preserved, and retrieved, and (4) how this information was used for the creation of historical earthquake catalogs. These conditions depend on various cultural-historical aspects that vary substantially over the entire Euro-Mediterranean region. Such a differentiation into areas of common completeness can be assumed for the territories covered by the national earthquake catalogs, because the usage of historical information in cataloging differs from country to country. Also, within a country there may be large differences in area (such as Italy with its considerable north-south spatial extent connected with different cultural-historical features), and consequently geographical variation exists in the completeness of historical information (Stucchi et al., 2004). We use the model developed by G. Grünthal et al. (unpublished manuscript, 2012) in connection with the European project SHARE as a suitable spatial subdivision of Europe into completeness zones. Figure 7 shows the four completeness zones considered in this study, which are part of the mentioned Euro-Mediterranean model. These examples have been selected to illustrate the applicability of the method to complicated cases.

An important aspect to be considered before a completeness study can be made is to check the declustering of the data. This study is restricted to declustered data because long-term earthquake databases are usually incomplete with regard to fore- and aftershocks in comparison to mainshocks. The declustering was performed using a method by Grünthal (1985); it is also described in Burkhard and Grünthal (2009), which is a more easily accessible source. Based on this approach, the time windows are calculated for foreshocks as

$$
\begin{aligned}
& d T_{f}\left(M_{\mathrm{w}}\right) \\
& \quad= \begin{cases}\exp \left(-4.77+\sqrt{0.62+17.32 M_{\mathrm{w}}}\right) & \text { if } M_{\mathrm{w}}<7.8 \\
\exp \left(6.44+0.055 M_{\mathrm{w}}\right) & \text { otherwise }\end{cases}
\end{aligned}
$$

and for aftershocks as

$$
\begin{aligned}
& d T_{a}\left(M_{\mathrm{w}}\right) \\
& \quad=\left\{\begin{array}{ll}
\exp \left(-3.95+\sqrt{0.62+17.32 M_{\mathrm{w}}}\right) & \text { if } M_{\mathrm{w}}<6.6 \\
\exp \left(6.44+0.055 M_{\mathrm{w}}\right) & \text { otherwise }
\end{array} .\right.
\end{aligned}
$$

The distance window is calculated as

$$
d R\left(M_{\mathrm{w}}\right)=\exp \left(1.77+\sqrt{0.037+1.02 M_{\mathrm{w}}}\right) .
$$

For a given mainshock, all smaller magnitude events within $d R$, occurring within the fore- or aftershock time windows, are considered dependent events and removed.

Completeness times have been estimated by applying the new method for different magnitude classes in each of the

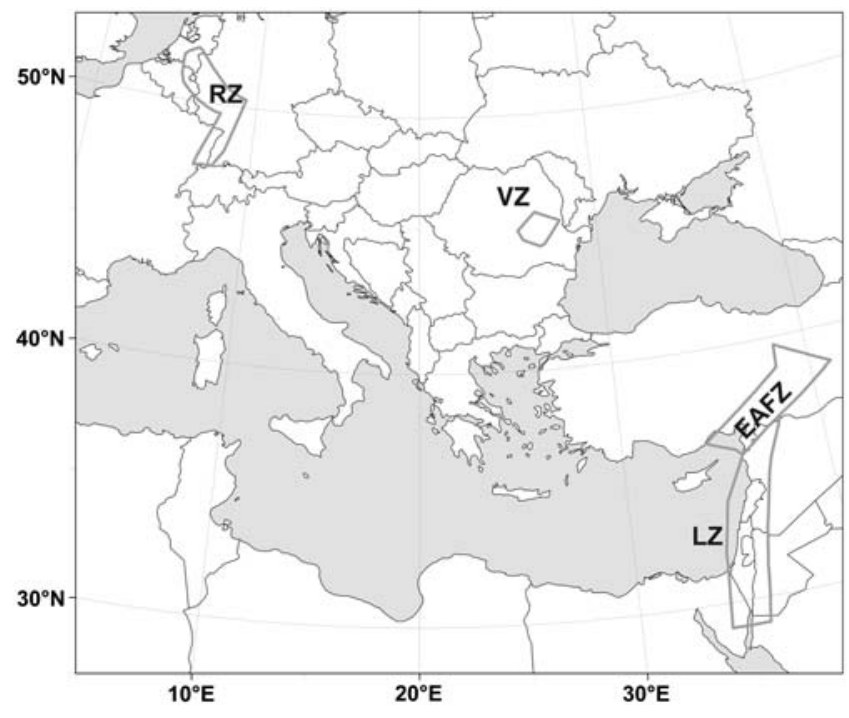

Figure 7. The four selected completeness zones in the EuroMediterranean region according to a model of completeness zones: the Levant zone (LZ), the Rhine zone (RZ), the East Anatolian fault zone (EAFZ), and the Vrancea zone (VZ).

four selected zones. Each magnitude class, $M$, contains earthquakes with magnitudes in an interval of half a magnitude unit $[M-0.25, M+0.25]$. The estimated completeness times are compared with those determined using the classical slope method.

\section{Application of the Method to Four Completeness Zones: The Levant, the Rhine Area, the East} Anatolian Fault Zone, and the Vrancea Region

From the historiographical point of view, the Levant zone (LZ) is a unique region and is defined as a specific zone for studying earthquake data completeness. The LZ is especially rich in historical sources from quite different cultures over more than two millennia. From the statistical point of view, the LZ is particularly interesting because the seismicity is notably nonstationary and follows a bimodal WeibullWeibull distribution (Hakimhashemi, 2010). The EMEC starts here in A.D. 300.

Tectonically the LZ is dominated by the Dead Sea fault zone, which is an active major left-lateral strike-slip fault zone, forming the boundary between the Arabian plate and the Sinai block (Mahmoud et al., 2005) as a part of the African plate. The Dead Sea fault zone stretches from the Red Sea in the south to the East Anatolian fault in the north (Fig. 7).

The completeness times of the seismicity data for the LZ are estimated using both the $F$-test and the NP test for magnitude classes from $M 4.5$ to 7.0, where each magnitude class $M$ contains events in the interval [ $M-0.25, M+0.25]$. Figures 8 and 9 display the completeness times of the different magnitude classes for declustered data using the $F$-test 
(a)

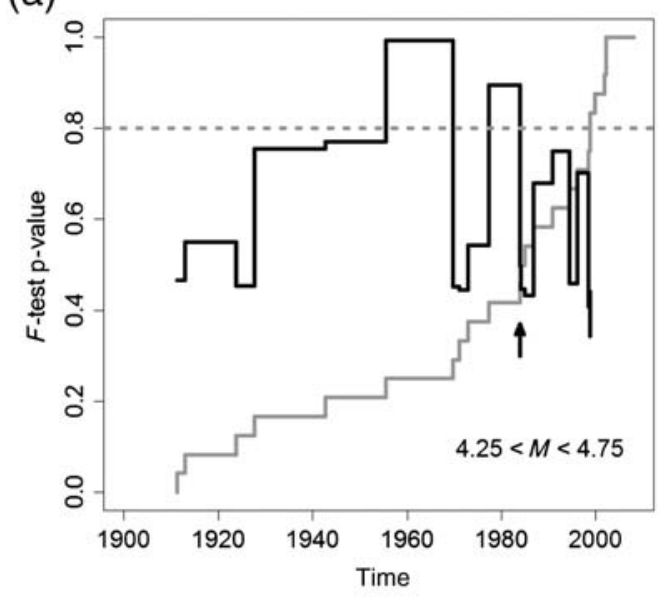

(c)

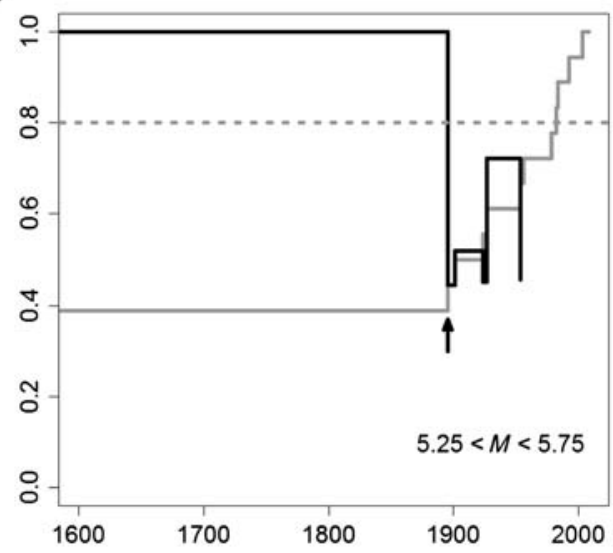

(e)

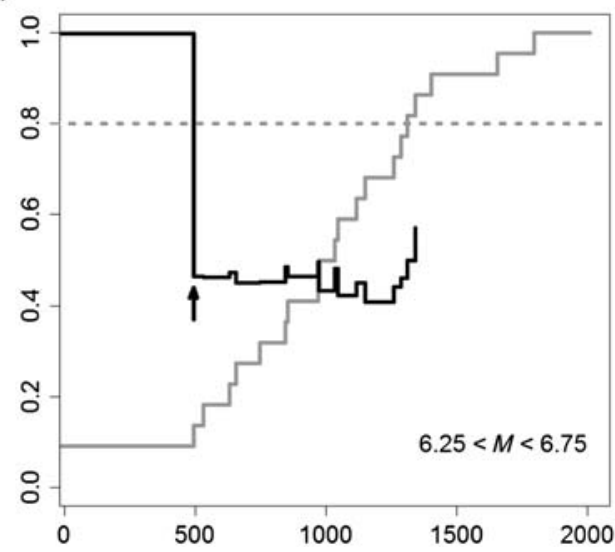

(b)

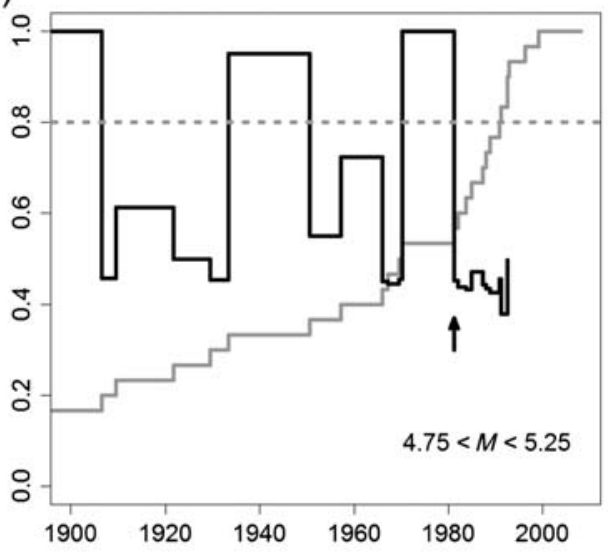

(d)

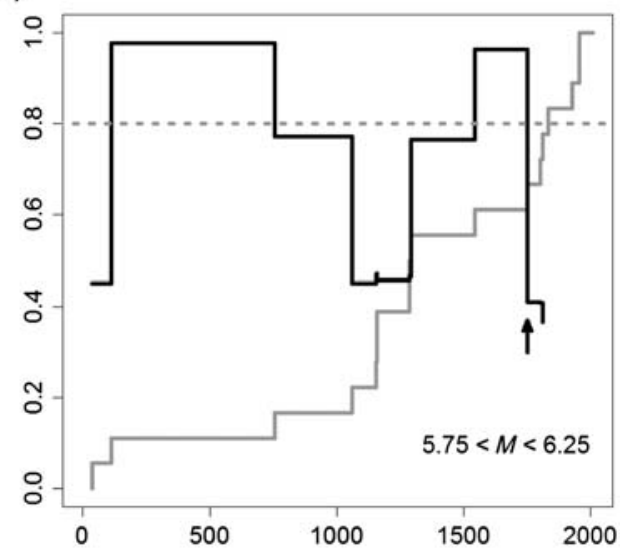

(f)

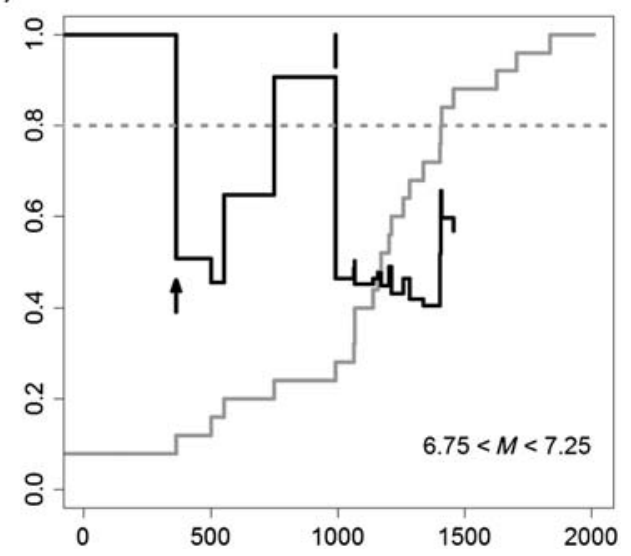

Figure 8. Completeness times of different magnitude classes using declustered seismicity data in the Levant zone (LZ). The p-values of $F$-tests of homogeneity of consecutive sample variances $V_{i}$ of earthquake interevent times (black lines) are compared with the normalized cumulative number of earthquakes versus corresponding earthquake occurrence times (gray lines). The gray dotted line presents the chosen level of confidence probability. The vertical axis represents the probability that the consecutive variances are different. Each magnitude class $M$ contains earthquakes in the interval $[M-0.25, M+0.25]$. The completeness times (arrows in the images) are selected according to the process explained in the text. The vertical bar in part (f) for magnitude class $M 7.0$ around year 990 is indicated as the first inverse time when the p-value reaches the confidence probability 0.8 . However, the completeness time of magnitude class $M 7.0$ is denoted by the arrow shortly before year 500, considering the completeness time of magnitude class $M 6.5$ and the rationale that the completeness time of a magnitude class cannot be later than that of a class of smaller magnitudes in the same area. 
(a)

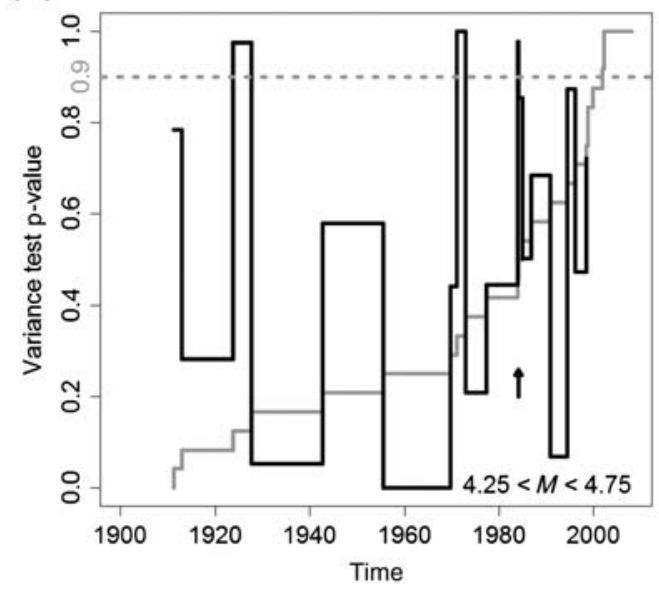

(c)

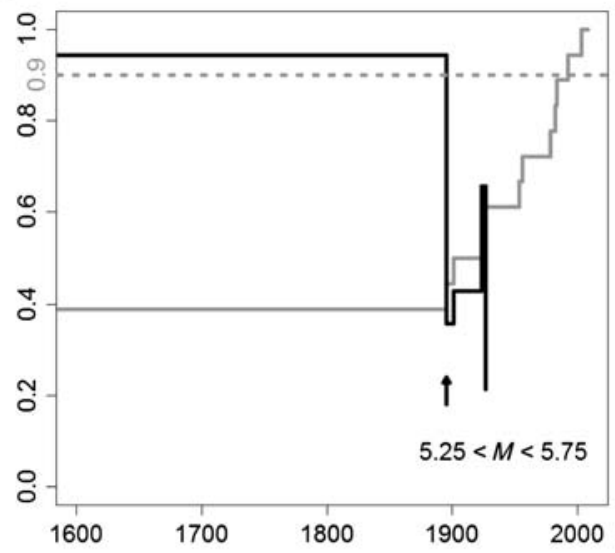

(e)

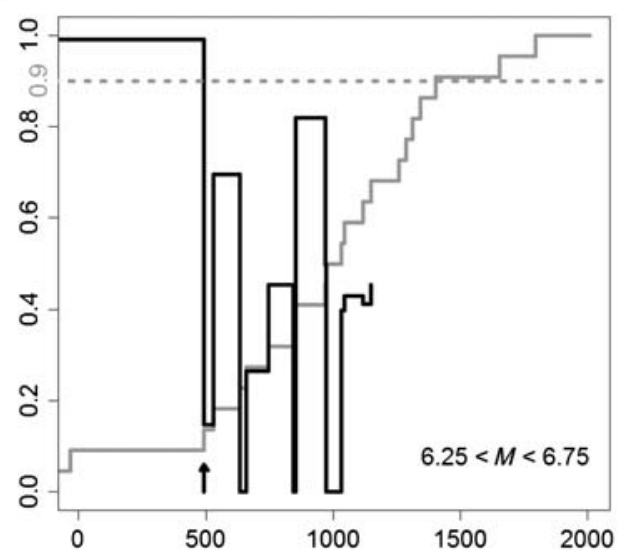

(b)

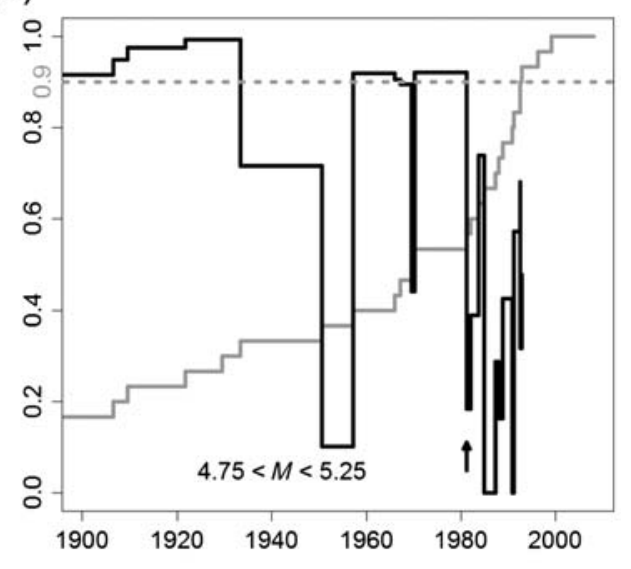

(d)

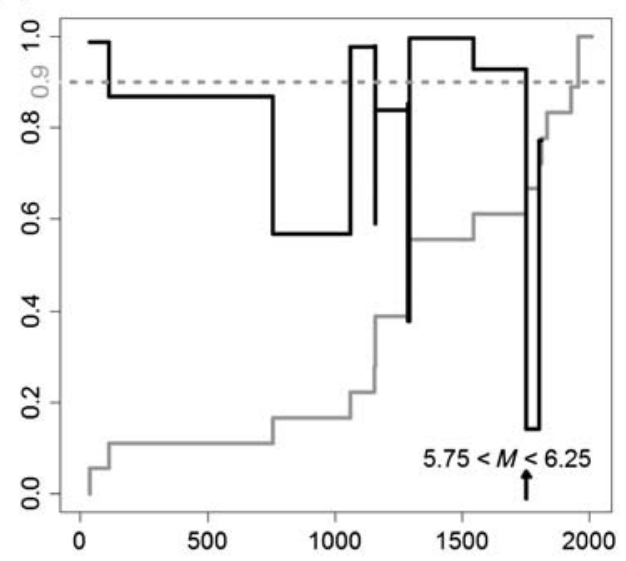

(f)

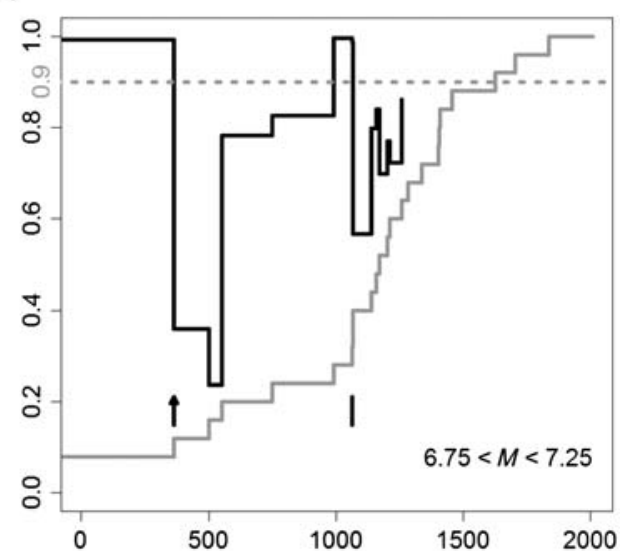

Figure 9. Same as Figure 8 but using p-values of the NP test of homogeneity of consecutive sample variances $V_{i}$ of earthquake interevent times (black lines). The detected completeness times are the same as for the $F$-test in Figure 8.

and the NP test respectively. Figures 8 and 9, respectively, show the p-values of the $F$-test and the p-values of the NP test of homogeneity of consecutive sample variances of interevent times together with the normalized cumulative number of earthquakes of various magnitude classes for declustered seismicity data in the LZ. For this and all following examples, the completeness time of each magnitude class is set as the time when the p-value is larger than the confidence 
Table 1

Completeness Times of Different Magnitude Classes for Declustered Seismicity Data Using Different Tests for the Selected Completeness Zones

\begin{tabular}{lllllllllc}
\hline & \multicolumn{7}{c}{ Magnitude Class $( \pm 0.25)$} \\
\cline { 2 - 9 } & 3.5 & 4.0 & 4.5 & 5.0 & 5.5 & 6.0 & 6.5 & 7.0 & 7.5 \\
\hline Levant Zone (LZ) & & & 1983 & 1981 & 1895 & 1752 & 493 & $493^{*}$ & $\dagger$ \\
New method $F$-test & & & 1983 & 1981 & 1895 & 1752 & 493 & $493^{\ddagger}$ & $\dagger$ \\
New method NP-test & & & 1985 & 1965 & 1890 & 1720 & 450 & 300 & 300 \\
Slope method & & & & & & & & & \\
Rhine Zone (RZ) & 1969 & 1870 & 1870 & 1827 & 1639 & $\dagger$ & & & \\
New method $F$-test & 1969 & $1870^{\S}$ & 1870 & 1827 & 857 & $\dagger$ & & & \\
New method NP-test & 1969 & 1870 & 1870 & 1820 & 1600 & 1250 & & & \\
Slope method & 1910 & 180 & & & & & & \\
East Anatolian Fault Zone (EAFZ) & & & & & & & \\
New method $F$-test & & & 1991 & 1976 & 1967 & 1927 & 1865 & 1821 & $\dagger$ \\
New method NP-test & & & 1995 & 1976 & 1967 & 1927 & 1788 & 1788 & $\dagger$ \\
Slope method & & 1975 & 1960 & 1930 & 1920 & 1820 & 1800 & 1800 \\
Vrancea Zone (VZ) & & & & & & & & \\
New method $F$-test & & & & & 1901 & 1777 & 1542 & 1037 \\
New method NP-test & & & & & & 1916 & 1777 & 1542 & 1169 \\
Slope method & & & & & & 1900 & 1750 & 1500 & 1400 \\
\hline
\end{tabular}

*Indicated is 990 (Fig. 8f); 493 is the completeness time of magnitude class 6.5 .

Data are too scarce; the new method cannot be applied.

FIndicated is 1062 (Fig. 9f); 493 is the completeness time of magnitude class 6.5.

${ }^{\S}$ Indicated is 1795 (Fig. 11b); 1870 is the completeness time of magnitude class 4.5 .

probability equal to 0.8 for the $F$-test and equal to 0.9 for the NP test, similar to the synthetic catalogs.

For the magnitude class $M 4.5$ (Figs. 8a and 9a), the p-values frequently change with time; however, with respect to the earthquake occurrence times between 1986 and 2006, the p-values are not significant enough (still under the corresponding confidence probability) and therefore, the corresponding occurrence times cannot be considered as complete. First in 1986 (when looking from current to the historical times), the corresponding p-values are larger than the corresponding confidence probabilities using the $F$-test and the NP test. This year can be defined as the start of sufficiently complete data of this magnitude class.

Table 1 shows completeness times of different magnitude classes, determined using both the NP test and the $F$-test, compared with the completeness times on the basis of the slope method for the LZ, as well as three other zones. The outcome of both tests and the slope method show a high degree of similarity. Considering the completeness time of magnitude class $M 6.5$ estimated by the two NP and $F$-test at A.D. 493, and the rationale that the completeness time of a class of stronger magnitudes cannot be earlier than of a class of smaller magnitudes, the completeness time of magnitude class $M 7.0$ will be similar and can be assumed to be in the same year.

For the LZ, the completeness times detected by both the $F$-test and the NP test are the same for all magnitude classes.

From the historiographical point of view, the Rhine zone (RZ) represents an area where German and French sources of information intersect. The Rhine area has the highest level of seismicity in Europe north of the Alpine chain. Tectonically active structures of graben formations, as well as strike-slip structures, generate the seismicity.

Figures 10 and 11 demonstrate the results of the $F$-test and of the NP test (corresponding p-values) of sample consecutive variances versus time for five magnitude classes, as well as the corresponding normalized cumulative number of earthquakes starting with the magnitude class $M 3.5$.

The completeness time of the magnitude class $M 4.0$, according to the NP test (Fig. 11b), is determined to be shortly before year 1800 (small vertical bar in the figure); however, as with the example of the LZ, it is reasonable to be considered as the completeness time of the magnitude class $M 4.5$ (i.e., year 1870; Fig. 11c). Another special case in the RZ is the magnitude class $M$ 5.5. The completeness time of this magnitude class as determined by the NP test is in year 857 (Fig. 11e) which is much earlier than the completeness time of year 1640 as determined by the $F$-test (Fig. 10e). There are two reasons for us to select year 1640 for the completeness time of the magnitude class $M$ 5.5. The first reason is that there is a large gap between two successive events in the corresponding data set; that is, the later one occurred in year 1640 whereas the previous one occurred in year 1080. The second reason is that the completeness time detected by the $F$-test is much closer to the one determined by the slope method. For other magnitude classes, Table 1 shows that the completeness times estimated by the two approaches are very similar.

The East Anatolian fault zone (EAFZ) is historiographically characterized as an intersecting region of Turkish/ 
(a)

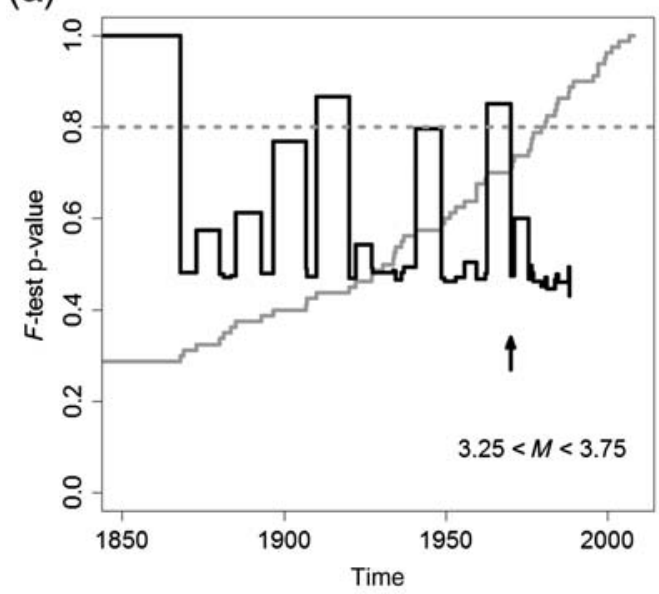

(c)

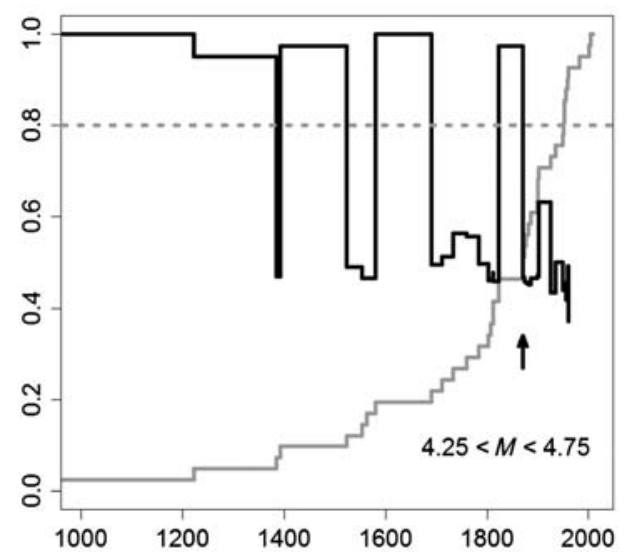

(b)

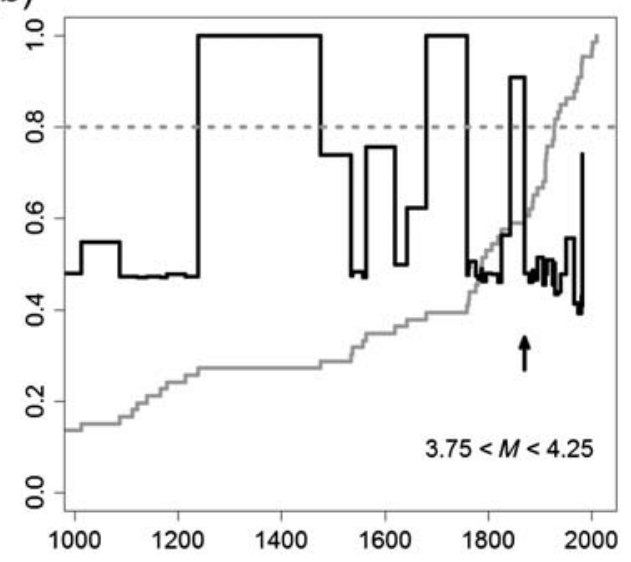

(d)

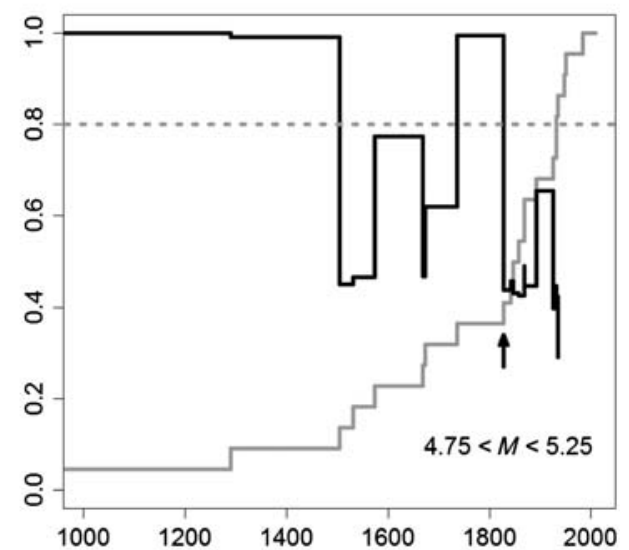

(e)

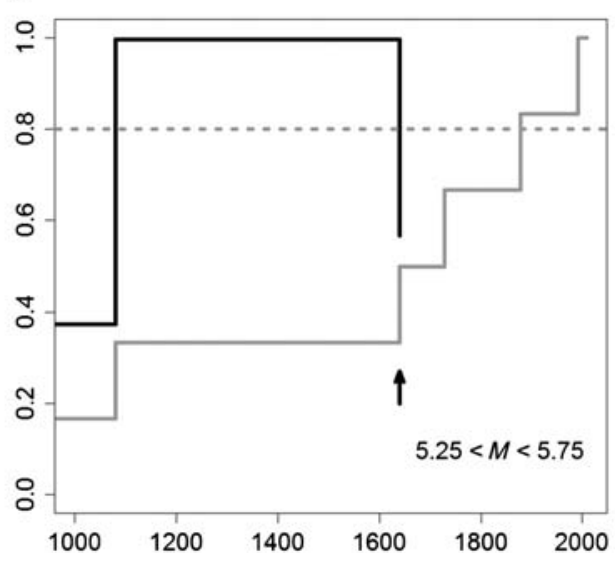

Figure 10. Same as Figure 8 ( $F$-test) for the declustered seismicity data in the Rhine zone (RZ).

Ottoman, Arabic, and Armenian influences. Tectonically, the seismically active EAFZ is the boundary between the Arabian plate and the Anatolian plate.

Figures 12 and 13 show the p-values of the $F$-test and of the NP test versus time, as well as the normalized cumulative number of events with time, for different magnitude classes. The graphs in Figures 12 and 13 confirm that the EAFZ represents a rather complicated case due to insufficient data in the magnitude classes $M_{\mathrm{w}} \geq 6.0$. The $\mathrm{p}$-value lines for the magnitude classes $M_{\mathrm{w}} \geq 6.0$ start (looking from current times to the past) with high p-values above the confidence probability. This effect is due to the insufficient number of events in the complete part of the data set. In such situations the method reaches its limits. 
(a)

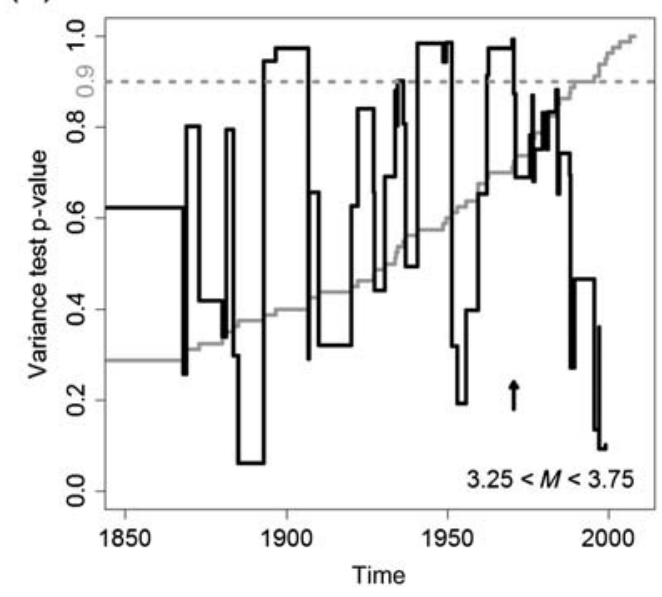

(c)

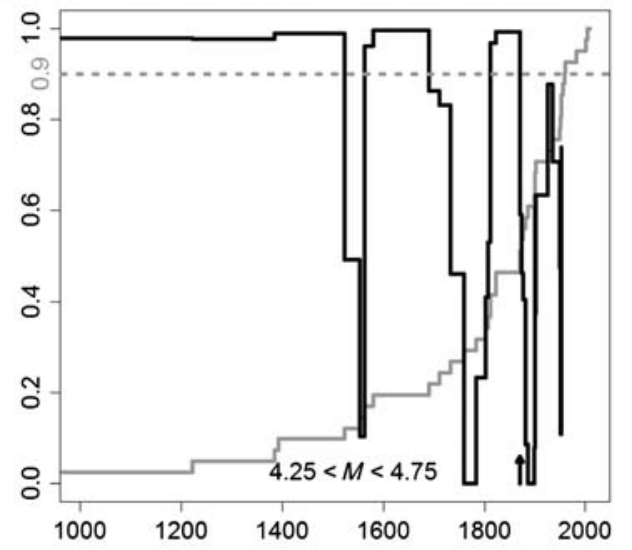

(b)

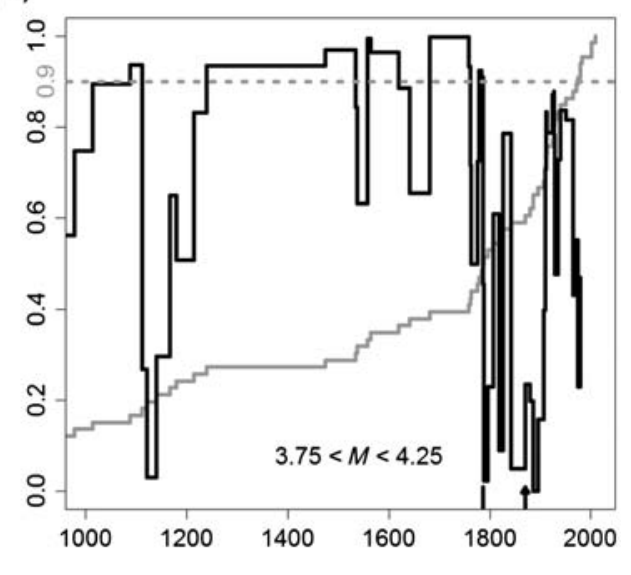

(d)

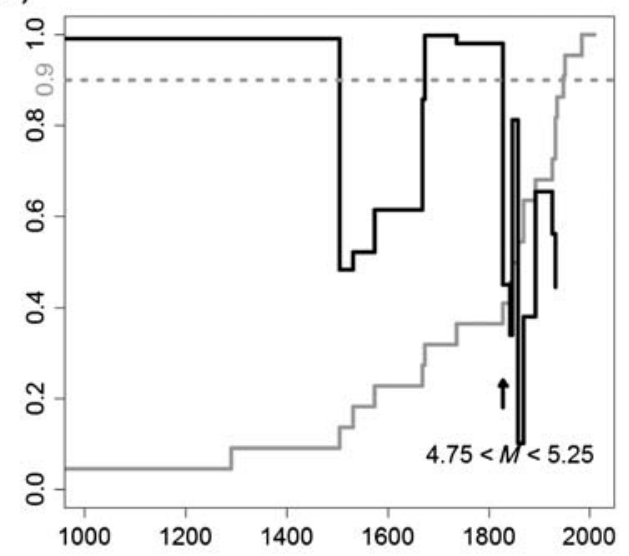

(e)

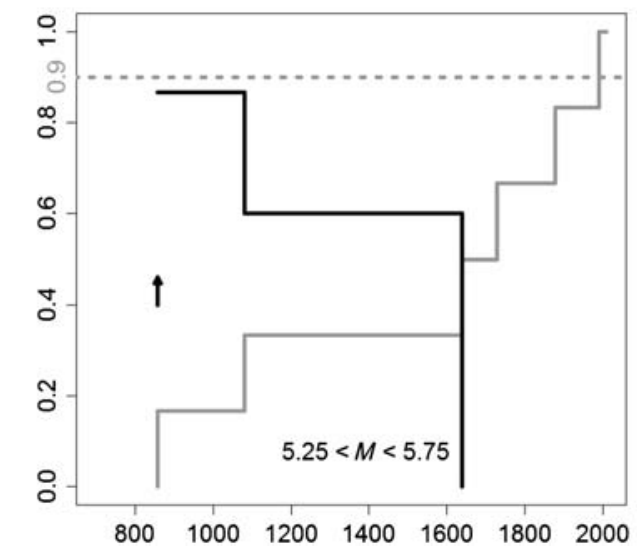

Figure 11. Same as Figure 9 (NP test) for the declustered seismicity data in the Rhine zone (RZ). The completeness time of magnitude class $M 4.0$ in (b) is indicated by the vertical bar to be shortly before year 1800; however, according to the completeness time of magnitude class $M 4.5$ in (c), it is considered as year 1870 (black arrow). The completeness time of magnitude class $M 5.5$ is indicated as the beginning of the catalog for this magnitude class. In this case the completeness time as indicated by the $F$-test is considered.

As shown in Table 1, the completeness times of magnitude classes $M_{\mathrm{w}} \geq 4.5$ derived by the new method are more conservative; that is, they appear later than the corresponding completeness times derived by the slope method. One reason for this may be that the completeness times by the new method are determined as a datum that is identified as the first event in the complete part of the data set. The Vrancea zone (VZ) is characterized by very localized intermediate-depth 
(a)

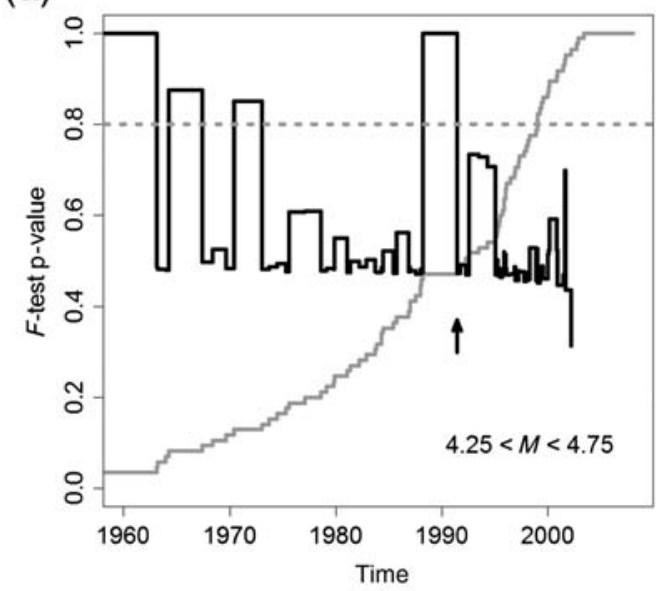

(c)

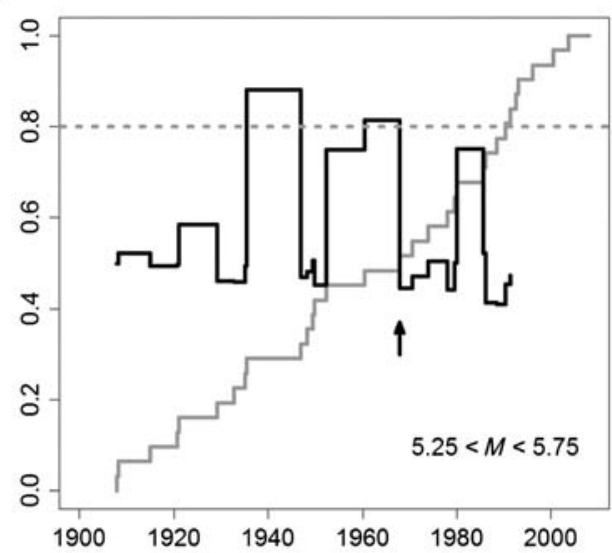

(e)

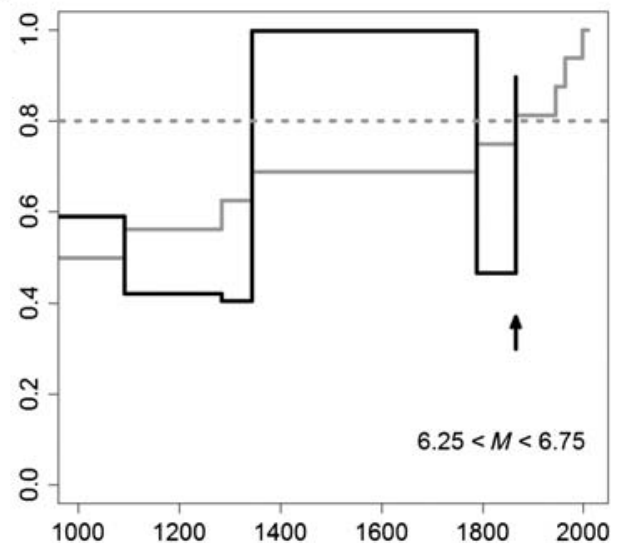

(b)

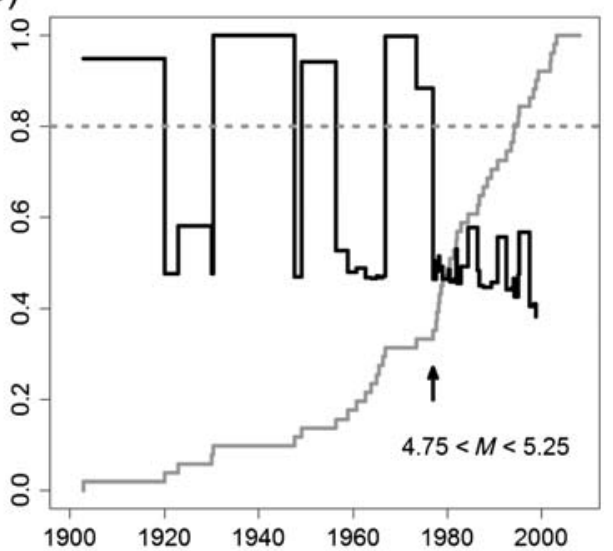

(d)

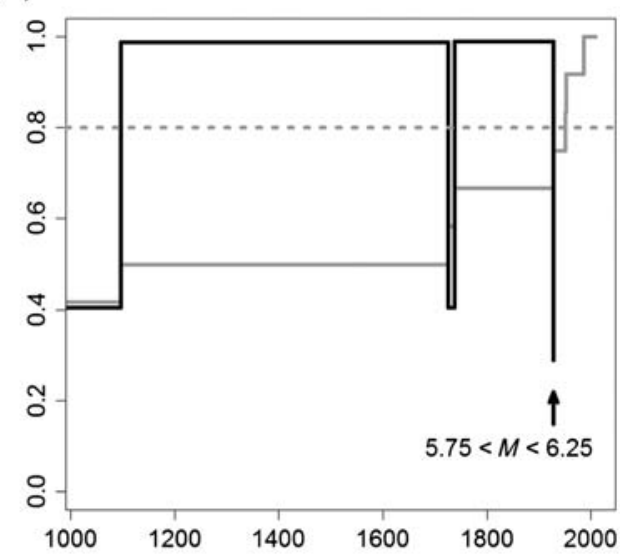

(f)

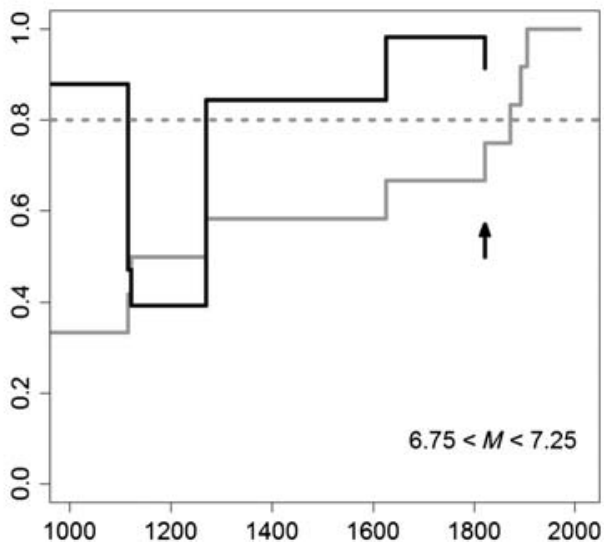

Figure 12. Same as Figure 8 ( $F$-test) for the declustered seismicity data in the East Anatolian fault zone (EAFZ).

seismicity, mostly in the range of $100-150 \mathrm{~km}$ where the strongest events occur. For such a deep source, the temporal detection threshold (and consequently the completeness time) will be different compared to shallow crustal seismicity. For this reason, the Vrancea area is identified as an individual zone. Tectonically it represents an old subduction zone in its very last phase of existence (Sperner et al., 2001;
Heidbach et al., 2007). The data sets for the magnitude classes $M_{\mathrm{w}} \leq 5.5$ show gaps and deficits. Therefore, we present only the results for the magnitude classes $M_{\mathrm{w}} \geq 6.0$. In Figure 14d, $F$-test p-values of the magnitude class $M 7.5$ never reach the confidence probability 0.8 . In other words, there is no significant change in the variance of different sets of earthquake interevent times. This means that the complete 
(a)

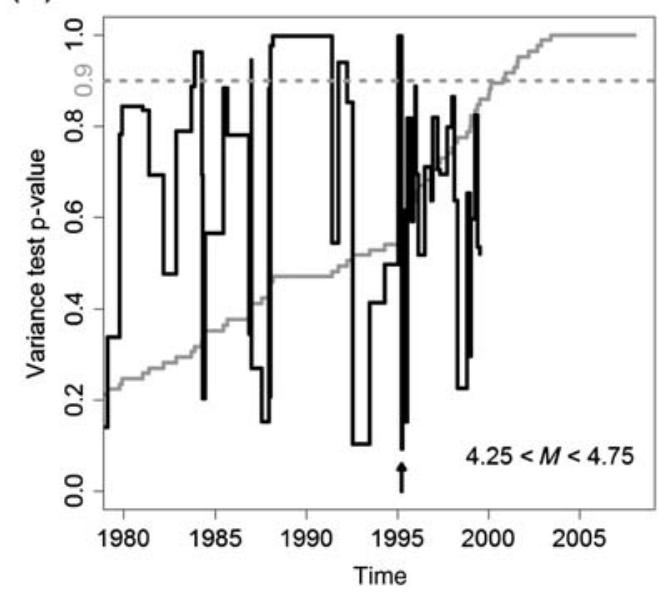

(c)

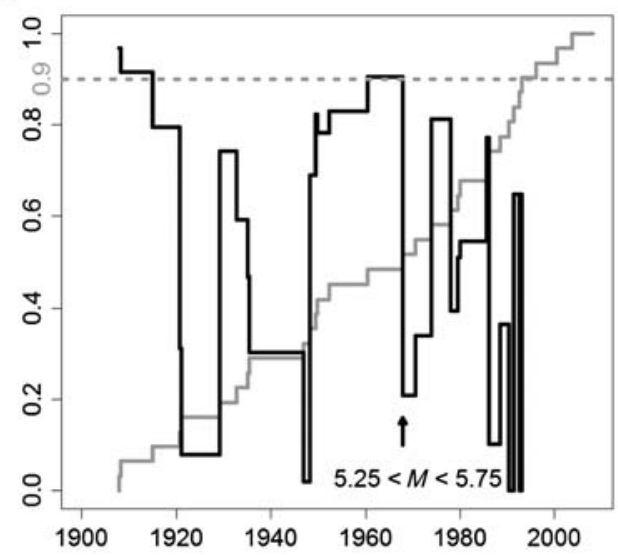

(e)

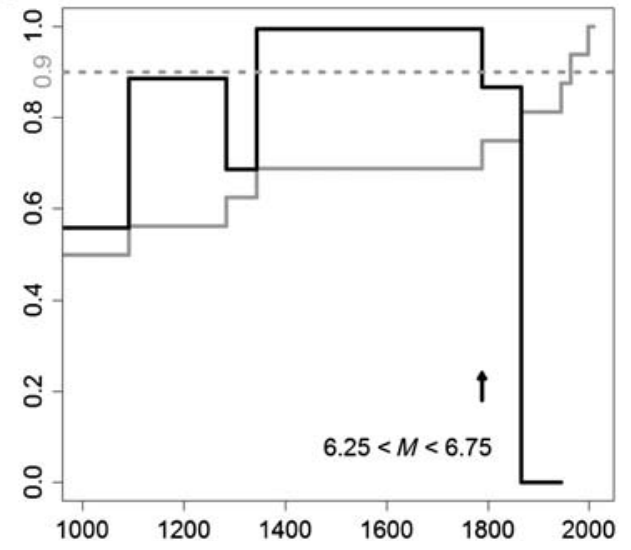

(b)

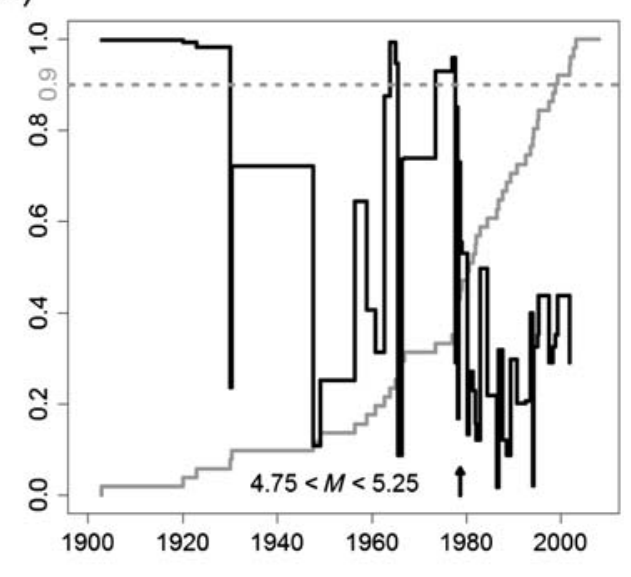

(d)

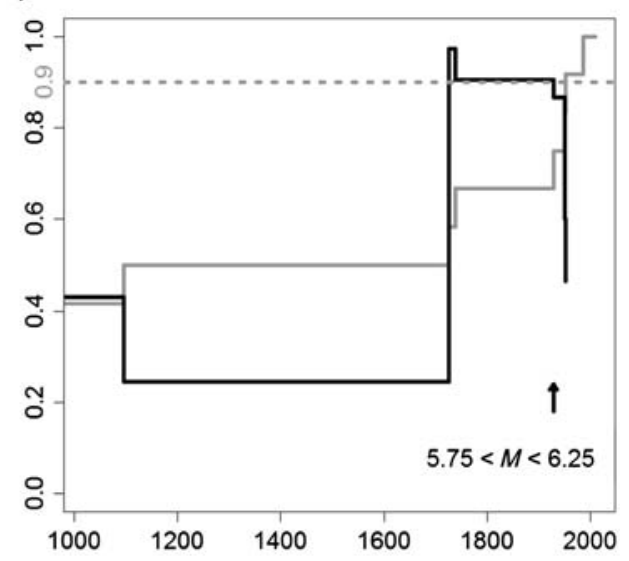

(f)

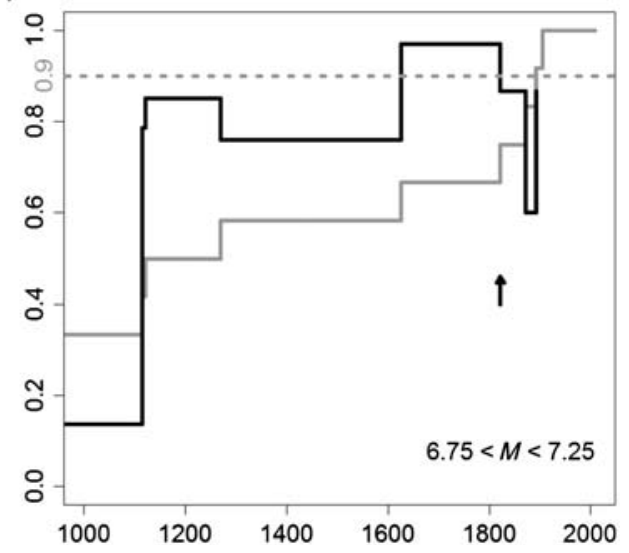

Figure 13. Same as Figure 9 (NP test) for the declustered seismicity data in the East Anatolian fault zone (EAFZ). The detected completeness time for magnitude class $M 4.5$ around year 1995 seems to be more reliable than the one detected by the $F$-test.

part of this data set covers the entire time range. Considering Figure $15 \mathrm{~d}$, the completeness time of the magnitude class $M 7.5$ starts from the second event in the corresponding data set, which is also close to the completeness time detected by the $F$-test. Table 1 illustrates a fairly good correspondence of the two methods.

\section{Conclusions}

The scarce number of large earthquakes in long-term seismicity data makes it necessary to use as much reliable information as possible. The new statistical method developed to estimate completeness times of historical catalogs 
(a)

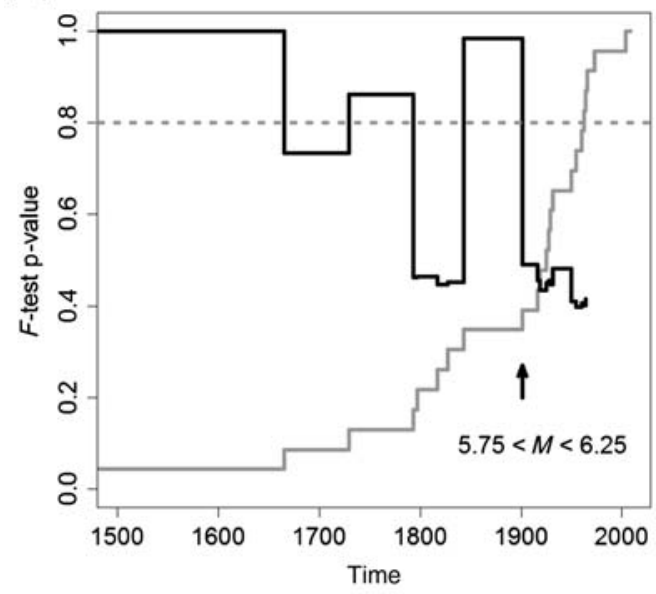

(c)

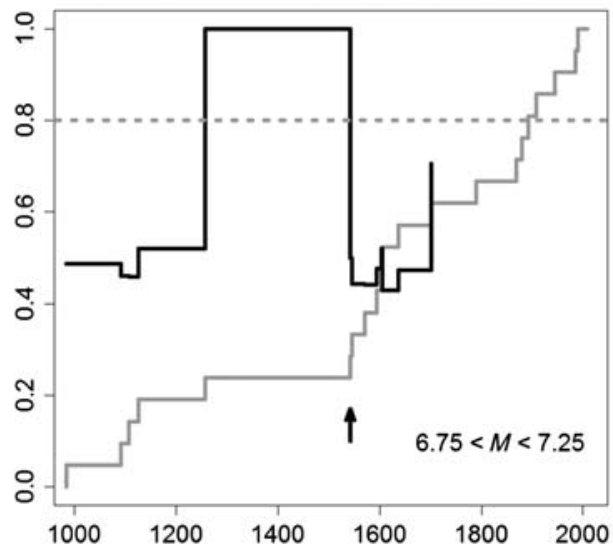

(b)

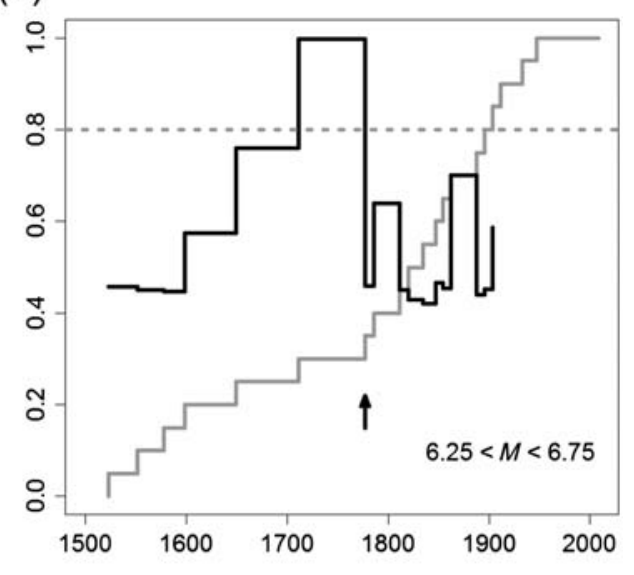

(d)

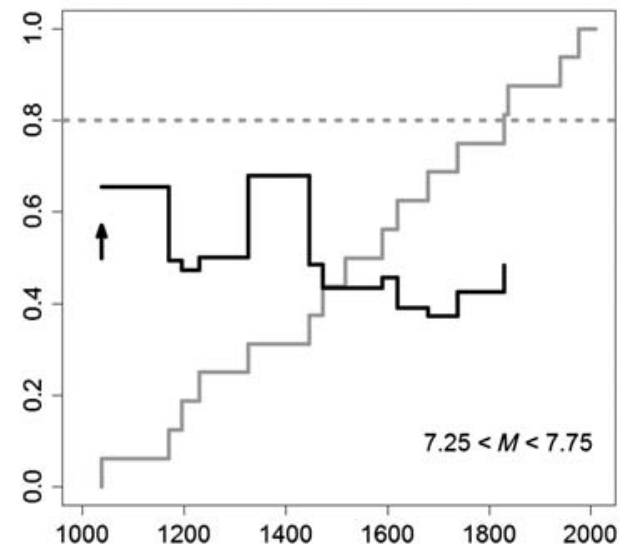

Figure 14. Same as Figure 8 ( $F$-test) for the declustered seismicity data in the Vrancea zone (VZ). The data sets corresponding to the classes with $M_{\mathrm{w}} \leq 5.5$ show gaps and deficits. Therefore, only the results for the classes with $M_{\mathrm{w}} \geq 6.0$ are represented.

for long-term seismicity data is simple and has been shown to be a reliable method. Previous statistical methods to determine the catalog completeness consider either a predefined stationary pattern (Poisson process) for the seismicity rate or a specific distribution. The new method avoids these presumptions so therefore is appropriate for both stationary and nonstationary seismicity. It is simple to apply, as the only parameter used for determining the completeness time is the variance of earthquake interevent times. The method explores the variances of different sets of interevent times and determines the completeness time as the time when the successive variances are significantly different. These differences are not always easily distinguishable. In these cases, an NP test as well as an $F$-test are used to detect the significant differences between consecutive variances.

The statistical tests on the basis of synthetic catalogs show an acceptable level of reliability of the method for stationary (Poisson process, exponentially distributed), nonstationary (gamma or lognormal distributed), and even clustered (bimodal Weibull-Weibull distributed) seismicity data. The synthetic tests show a very good convergence in spite of the automatic selection procedure of completeness times.

The method has also been applied to real long-term catalog data. We used both the NP test and the $F$-test p-values to identify the completeness time as the time when the p-value reaches the selected confidence probability. In general, the completeness times detected by both tests are very similar. The results of the synthetic and real catalogs confirm that the NP test is generally more sensitive in comparison with the $F$-test. The selected examples are in general not amenable to completeness time analysis. For these data sets, the results of the new method are generally in good agreement with the results obtained by the classical slope method. However, in some cases the new method tends to lead to more conservative estimates.

The method requires a sufficient number of data, depending on the data quality. In the case of too scarce events, the method may reach its limits. For example, the completeness times of magnitude classes $M_{\mathrm{w}} \geq 6.0$ in the EAFZ start almost at the end of the corresponding data sets (Figs. 12 and 13). Here the complete parts include only very 
(a)

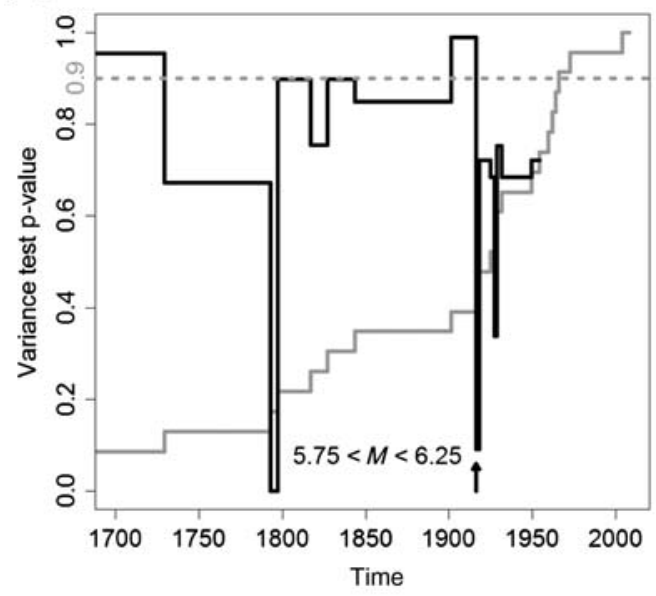

(c)

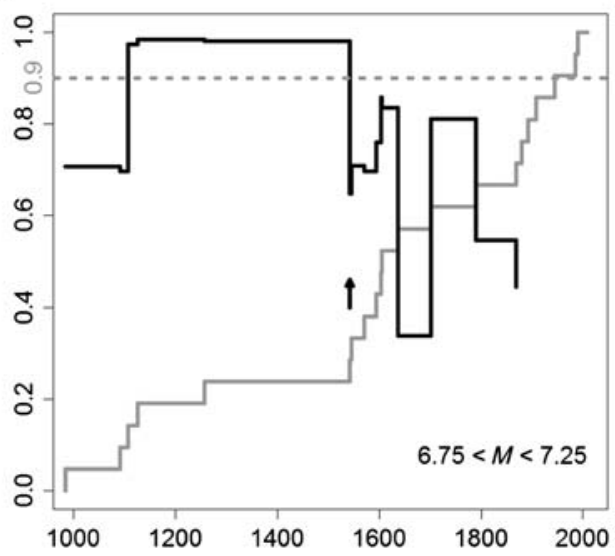

(b)

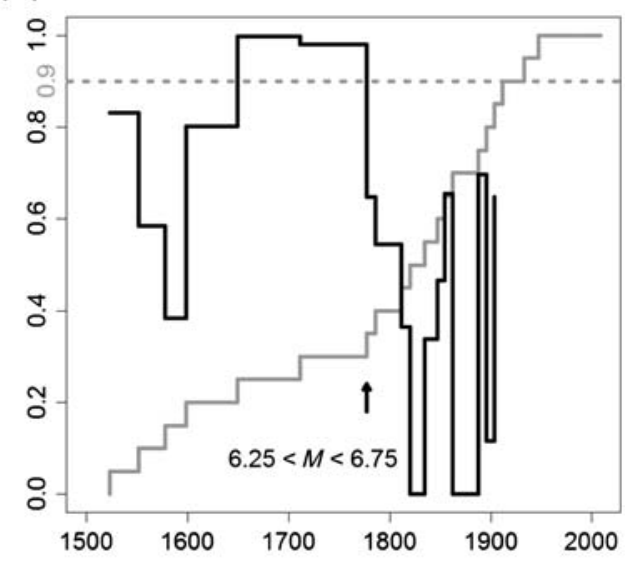

(d)

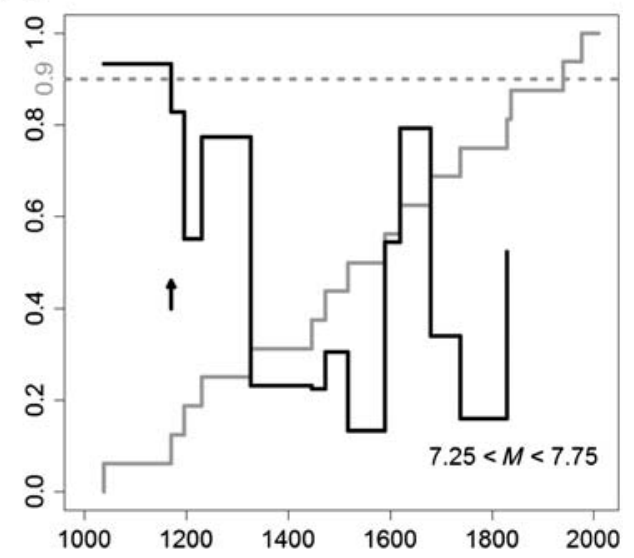

Figure 15. Same as Figure 9 (NP test) for the declustered seismicity data in the Vrancea zone (VZ).

few events. In such cases, the method should be used with care. Therefore, we suggest a combination of the results of the new method with those of the slope method as well as historiographical information.

\section{Data and Resources}

We used the seismicity data extracted from EuropeanMediterranean Earthquake Catalogue (EMEC; Grünthal and Wahlström, 2012).

\section{Acknowledgments}

This study has been carried out under the framework of the DEad Sea Integrated REsearch project (DESIRE), founded by the Deutsche Forschungsgemeinschaft (German Research Foundation). We appreciate this support and the support from the German Research Centre for Geosciences, GFZ Potsdam. We also thank Jean-Pierre Stockis and Rutger Wahlström for their valuable comments.

\section{References}

Albarello, D., R. Camassi, and A. Rebez (2001). Detection of space and time heterogeneity in the completeness level of a seismic catalogue by a statistical approach: An application to the Italian area, Bull. Seismol. Soc. Am. 91, no. 6, 1694-1703.

Amorese, D. (2007). Applying a change-point detection method on frequency-magnitude distributions, Bull. Seismol. Soc. Am. 97, no. $5,1742-1749$.

Båth, M. (1983). Earthquake data analysis: An example from Sweden, Earth Sci. Rev. 19, 181-303.

Burkhard, M., and G. Grünthal (2009). Seismic source zone characterization for the seismic hazard assessment project PEGASOS by the Expert Group 2 (EG1b), Eclogae Geol. Helv. 102, 149-188.

Cao, A. M., and S. S. Gao (2002). Temporal variations of seismic $b$-values beneath northeastern Japan island arc, Geophys. Res. Lett. 29, no. 9, 48-1-48-3.

Caputo, M., and D. Postpischl (1974). Contour mapping of seismic areas by numerical filtering and geological implications, Ann. Geofis. 27, 619-640.

Fisher, R. (1918). The correlation between relatives on the supposition of Mendelian inheritance, Phil. Trans. Roy. Soc. Edinburgh 52, 399-433.

Grünthal, G. (1985). The up-dated earthquake catalogue for the German Democratic Republic and adjacent areas-Statistical data characteristics and conclusions for hazard assessment, 3rd International Symposium on the Analysis of Seismicity and Seismic Risk, Liblice/Czechoslovakia, 17-22 June 1985, Proceeding Vol. I, 19-25.

Grünthal, G., and R. Wahlström (2012). The European-Mediterranean Earthquake Catalogue (EMEC) for the last millennium, J. Seismol. 16, no. 3, 535-570. 
Grünthal, G., D. Mayer-Rosa, and W. Lenhardt (1998). Abschätzung der Erdbebengefährdung für die D-A-CH-Staaten-Deutschland, Österreich, Schweiz, Bautechnik 75, no. 10, 753-767.

Grünthal, G., R. Wahlström, and D. Stromeyer (2009). The unified catalogue of earthquakes in central, northern, and northwestern Europe (CENEC) - Updated and expanded to the last millennium, J. Seismol. 13, no. 4, 517-541.

Hakimhashemi, A. H. (2010). Time-dependent occurrence rates of large earthquakes in the Dead Sea fault zone and applications to probabilistic seismic hazard assessments, Ph.D. Thesis, Potsdam University.

Heidbach, O., P. Ledermann, D. Kurfess, G. Peters, T. Buchmann, L. Matenco, M. Negut, B. Sperner, B. Müller, A. Nuckelt, and G. Schmitt (2007). Attached or not attached: Slab dynamics beneath Vrancea, Romania, International Symposium on Strong Vrancea Earthquakes and Risk Mitigation, 4-6 October 2007, Bucharest, Romania, 3-20.

Joanes, D. N., and C. A. Gill (1998). Comparing measures of sample skewness and kurtosis, J. Roy. Stat. Soc. D: Statistician 47, no. 1, 183-189.

Lehmann, E. L. (2006). Nonparametrics: Statistical Methods Based on Ranks, McGraw-Hill, New York.

Mahmoud, S., R. Reilinger, S. McClusky, P. Vernant, and A. Tealeb (2005) GPS evidence for northward motion of the Sinai block: Implications for E. Mediterranean tectonics, Earth Planet. Sci. Lett. 238, no. 1-2, 217-224.

Mignan, A., M. J. Werner, S. Wiemer, C.-C. Chen, and Y.-M. Wu (2011) Bayesian estimation of the spatially varying completeness magnitude of earthquake catalogs, Bull. Seismol. Soc. Am. 101, no. 3, 1371-1385.

Mulargia, F., and S. Tinti (1985). Seismic sample area defined from incomplete catalogs: An application to the Italian territory, Phys. Earth Planet. In. 40, 273-300.

Mulargia, F., P. Gasperini, and S. Tinti (1987a). A procedure to identify objectively active seismotectonic structures, Boll. Geofis. Teor. Appl. 29, no. 114, 147-164.

Mulargia, F., P. Gasperini, and S. Tinti (1987b). Contour mapping of Italian seismicity, Tectonophysics 142, 203-216.

Navidi, W. (2006). Statistics for Engineers and Scientists, McGraw-Hill, New York, 14 pp.

Rotondi, R., and E. Garavaglia (2002). Statistical analysis of the completeness of a seismic catalogue, Nat. Haz. 25, no. 3, 245-258.
Rotondi, R., F. Meroni, and G. Zonno (1994). A different intensity recording for reducing the uncertainty in its assessment: An application to the completeness analysis of earthquake catalogs, Nat. Haz. 10, 45-58

Siegel, S., and J. W. Tukey (1960). A nonparametric sum of ranks procedure for relative spread in unpaired samples, J. Am. Stat. Assoc. 55, no. 291, 429-445.

Sperner, B., F. P. Lorenz, K. P. Bonjer, S. Hettel, B. Müller, and F. Wenzel (2001). Slab break-off-abrupt cut or gradual detachment? New insights from the Vrancea region (SE Carpathians, Romania), Terra Nova 13, $172-179$.

Stepp, J. C. (1972). Analysis of the completeness of the earthquake sample in the Puget Sound area and its effect on statistical estimates of earthquake hazard, Proc. of the International Conf. on Microzonation for Safer Construction: Research and Application, Seattle, Washington 64, 1189-1207.

Stucchi, M., P. Albini, M. Mirto, and A. Rebez (2004). Assessing the completeness of Italian historical earthquake data, Ann. Geofis. 47 no. 2-3.

Tinti, S., and F. Mulargia (1985a). Completeness analysis of a seismic catalog, Ann. Geophys. 3, 407-414.

Tinti, S., and F. Mulargia (1985b). An improved method for the analysis of the completeness of a seismic catalog, Lettere al Nuovo Cimento $\mathbf{4 2}$, 21-27.

Wiemer, S., and M. Wyss (2000). Minimum magnitude of complete reporting in earthquake catalogs: Examples from Alaska, the western United States, and Japan, Bull. Seismol. Soc. Am. 90, 859-869.

Woessner, J., and S. Wiemer (2005). Assessing the quality of earthquake catalogues: Estimating the magnitude of completeness and its uncertainty, Bull. Seismol. Soc. Am. 95, no. 2, 684-698.

Helmholtz Centre Potsdam

GFZ German Research Centre for Geosciences

Section 2.6, Seismic Hazard and Stress Field

Telegrafenberg

D-14473 Potsdam, Germany

hakim@gfz-potsdam.de

ggrue@gfz-potsdam.de

Manuscript received 4 November 2011 